\title{
JOINT VENTURES Y ESPECIALIZACIÓN PRODUCTIVA EN LA INDUSTRIA DEL VINO EN CHILE ${ }^{1}$
}

\author{
R. Javier Bustos, Julio Peña Torres \\ y Manuel Willington
}

En este trabajo se analizan los diferentes patrones de especialización productiva que han seguido inversionistas extranjeros en la industria del vino en Chile; en particular, el efecto de la estructura de propiedad que la firma escoge para su nuevo negocio vitivinícola. Existe evidencia que viñas organizadas como joint venture (JV) tienen mayor participación en los segmentos de vinos exportados con mayor valor unitario que las viñas que son filiales de inversionistas extranjeros. La hipótesis central bajo estudio es que para inversionistas extranjeros con producción más especializada en vinos de mayor

R. JAVIER Bustos. Economista, área de regulación económica, Comisión Nacional de Energía.Email: jbustos@cne.cl.

Julio Peña Torres. Profesor Adjunto en la Facultad de Economía y Negocios, ILADES-Universidad Alberto Hurtado.Email: jpena@uahurtado.cl.

Manuel Willington. Profesor Auxiliar en la Facultad de Economía y Negocios, ILADES-Universidad Alberto Hurtado.Email: mwilling@uahurtado.cl.

${ }^{1}$ La recopilación de información relevante no habría sido posible sin la colaboración de Graciela Moguillansky y Sebastián Vergara, ambos miembros de la División de Desarrollo Productivo y Empresarial de CEPAL (Santiago, Chile). Igualmente importante ha sido la cooperación de la Corporación Chilena del Vino, así como de gerentes y enólogos de las viñas entrevistadas. Agradecemos los comentarios recibidos en el ciclo de seminarios del Departamento de Economía de la Universidad Alberto Hurtado. El contenido del trabajo es de exclusiva responsabilidad de los autores.

Estudios Públicos, 109 (verano 2008). 
valor, acuerdos de 'propiedad compartida' o JV pueden ser un mecanismo eficiente para incentivar las inversiones y esfuerzos productivos requeridos por tal tipo de producción. Las razones analizadas se refieren fundamentalmente al diseño de incentivos contractuales eficientes.

\section{Introducción}

$\mathrm{H}_{\mathrm{a}}$ acia fines de los años ochenta, el 85\% del total de vino exportado en el mundo provenía de cuatro países productores: Francia, Italia, España y Portugal. Una década después la participación de este grupo no sobrepasaba el 70\% (The Economist, 1999). Lo anterior ha sido producto del acelerado desarrollo exportador experimentado a partir de la década de los 90 por varios otros países productores; entre éstos, Australia, Estados Unidos (California), Chile, Nueva Zelanda, Sudáfrica y Argentina.

En el caso de Chile, desde inicios de los 80 se ha producido un creciente desarrollo exportador de vinos. En el año 1984 sólo 2\% de la producción se destinaba a mercados externos, mientras que el 2006 tal proporción bordea el 83\%. Durante el año 2006 las exportaciones fueron alrededor de US\$1.000 millones, en tanto el consumo interno alcanzó en torno a US\$200 millones. Asimismo, los volúmenes totales de producción se triplicaron durante la década de los noventa; mientras que el área con cultivos de vinos se ha duplicado desde mediados de los 90, alcanzado actualmente las 100.000 hectáreas.

Como parte de este proceso de crecimiento han surgido estrategias para diversificar y especializar la producción, incluyendo esfuerzos productivos para vender vinos de mayor valor en los mercados internacionales. En esto último la inversión extranjera ha jugado un rol relevante. Alrededor de un tercio de las exportaciones de vinos más caros (categoría 'ícono’) lo realizan viñas con participación societaria extranjera, mientras que su participación se reduce a menos del $2 \%$ en segmentos de vinos de menor valor (categorías ‘de mesa’ y ‘corriente’).

En este trabajo se analizan diferencias en los patrones de especialización productiva que han seguido inversionistas extranjeros en la industria del vino en Chile. En particular, se analiza de qué forma y por qué el patrón de especialización productiva puede condicionar la elección que hace el inversionista extranjero respecto de la estructura de propiedad de la firma que implementará el nuevo negocio vitivinícola. 
De un total de 265 viñas productoras en Chile, según datos para el año 2004, cuarenta de ellas tenían participación extranjera, fuese en la forma de un joint venture (JV) con alguna viña local, o bien a través de una empresa filial (i.e., 100\% de propiedad) del inversionista extranjero. En la actualidad, este grupo de cuarenta viñas produce algo más del 15\% del valor (FOB) total exportado de vinos (Nuevos Mundos: Compendio Vitivinícola de Chile, 2006).

Dentro del grupo de viñas con inversión extranjera en Chile existen diferencias en sus patrones de especialización productiva. Si se aproxima lo último por el valor promedio de los vinos producidos, se constata que el precio promedio de exportación logrado por viñas organizadas como JV fue 4,9 US\$/botella durante el periodo 1998-2004, mientras que el promedio del grupo de viñas organizadas como filiales fue 2,1 US\$/botella. En ese mismo periodo, el valor promedio del total de vinos exportados desde Chile fue 1,6 US\$/botella (Nuevos Mundos, 2004).

En los nichos de mercado con precios (FOB) de exportación por sobre 4,2 US\$/botella (categoría de 'vinos íconos'), en los años 2002-2004 las viñas tipo JV controlaban $25-30 \%$ de este segmento de mercado; en tanto que las viñas organizadas como filiales tenían una participación grupal de 8-10\%. Lo anterior permite ilustrar un punto más general: el grupo de viñas organizadas como JV presenta mayores participaciones de mercado en los segmentos de mayor valor unitario de los vinos exportados desde Chile, en relación al grupo de filiales de inversionistas extranjeros. Lo opuesto ocurre con las participaciones de uno y otro grupo de viñas en los segmentos con más bajos precios de exportación.

El uso de JV para producir vinos de mayor valor no parece ocurrir exclusivamente en Chile. Uno de los JV más antiguos es el que llevan a cabo, a partir de 1979, Robert Mondavi (la segunda firma en importancia en EE.UU.) y el Chateau Mouton Rothschild (una de las viñas más tradicionales de Francia), denominado Opus One y cuya producción se localiza en California ${ }^{2}$. Dentro de las firmas extranjeras que han invertido en Chile, la tradicional firma francesa Domaines Barons de Rothschild (en el negocio vitivinícola desde 1868) tiene tres JV: uno en Chile (Viña Los Vascos), otro en Portugal (Quinta Do Carmo) y otro en Argentina (Bodegas Caro) ${ }^{3}$.

Precisamente en Argentina en los últimos años, de manera paralela a la creciente especialización en vinos de mayor calidad que ha experimentado la industria (los vinos de mesa, de constituir el $44 \%$ del total de exportaciones en 1996, han pasado a representar el $14 \%$ en el año 2003, según

\footnotetext{
${ }^{2}$ http://www.mondavi.com.

${ }^{3}$ http://www.latife.com.
} 
www.areadelvino.com e Instituto Nacional de Vitivinicultura), se ha verificado un uso creciente de la figura de joint ventures con viñas extranjeras ${ }^{4}$ (Azpiazu y Basualdo, 2003; Bajda, 2000; Aguirre, 2001).

Previo a continuar, es importante precisar qué significa un acuerdo contractual tipo joint venture. Un JV comparte una serie de características con otros mecanismos de emprendimiento conjunto entre dos o más partes; por ejemplo, que exista un objetivo en común entre las partes y también reglas explícitas de reparto de las ganancias resultantes del negocio conjunto. Sin embargo, lo peculiar al concepto de JV es que en este tipo de contrato las partes acuerdan derechos de 'propiedad conjunta' sobre (al menos parte de) los activos requeridos por tal emprendimiento. Según la definición de joint venture en el Black's Law Dictionary (Garner, 2004), 'propiedad conjunta' implica que cada parte posee igual derecho de control para decidir qué usos dar, más allá de los estipulados expresamente por ley o contrato, a los activos bajo propiedad conjunta. Así, cada parte en un JV posee 'derecho de veto' sobre los usos posibles de dar a los activos bajo propiedad común. En este trabajo se desarrollan argumentos para explicar la preferencia relativa de inversionistas extranjeros por estructuras de propiedad tipo $\mathrm{JV}$, en desmedro de la opción de recurrir a una empresa filial, cuando se busca una especialización en vinos de mayor valor. Con base en literatura económica asociada a la 'Nueva Teoría de la Firma' (e.g., Grossman y Hart, 1986; Hart y Moore, 1990; Holmstrom y Roberts, 1998; Cai, 2003), los argumentos se centran en analizar los tipos de activos y esfuerzos productivos requeridos por disímiles patrones de especialización productiva.

\footnotetext{
${ }^{4}$ Sin embargo, si se comparan las estructuras de propiedad de viñas en Chile y Argentina, concentrándose en los casos con participación de inversionistas extranjeros, queda en evidencia el mayor uso de JV en Chile. Durante el periodo 1988-2003, más del $50 \%$ de la inversión extranjera en la industria vitivinícola de Chile se realizó vía JV (Nuevos Mundos, 2004). En Argentina esta proporción ha bordeado el 14\%, considerando el periodo 1992-2002. Los primeros JV vitivinícolas en Argentina aparecen entre los años 1999-2000. Por ejemplo, Bodegas Caro (JV entre Domaines Barons de Rothschild y la viña argentina Catena) comenzó a operar en 1999 (Azpiazu y Basualdo, 2003).

${ }^{5}$ El rezago relativo de Argentina respecto a Chile, en cuanto a la producción de vinos de alta calidad, se explicaría por razones particulares a la política vitivinícola argentina. El marco institucional vigente en Argentina favoreció mediante diversas regulaciones, a partir de la década de los 70 y por un número importante de años, la plantación de uvas comunes y la producción de vinos de bajo valor (destinados al consumo interno). Vía estímulos impositivos y financieros, se subsidiaba la incorporación de nuevas tierras al cultivo de este tipo de uvas y producción vitivinícola. Así, la plantación de vides comunes, de alto rendimiento cuantitativo y baja calidad enológica, aumentó significativamente la oferta potencial de vinos de mesa y corrientes (Mo, 1979), incentivando a la vez el continuar privilegiando la producción a gran escala, sacrificando calidad por cantidad (Azpiazu y Basualdo, 2003).
} 
La hipótesis central en estudio es que para inversionistas extranjeros con producción especializada en vinos de valor elevado, acuerdos tipo JV pueden constituir un mecanismo eficiente para incentivar las inversiones y esfuerzos productivos requeridos por tal tipo de producción. Así, las justificaciones analizadas se refieren al diseño de incentivos contractuales. Por un lado, incentivos para realizar determinados tipos de inversión. Por otro, incentivos para resolver problemas de agencia con origen en asimetrías de información entre las contrapartes en transacción. Y en tercer lugar, incentivos para favorecer el traspaso de conocimientos demasiado costosos de intercambiar en forma directa.

Este trabajo no pretende ofrecer un análisis exhaustivo del conjunto de posibles motivaciones para elegir acuerdos tipo JV como estructura de propiedad para emprendimientos vitivinícolas. El interés se centra en analizar problemas de incentivos y costos de transacción asociados principalmente a las fases de cultivo y de elaboración del vino en bodega. Lo anterior, dado que el foco de análisis está en la elección de la estructura de propiedad que realiza el inversionista extranjero ${ }^{6}$. Asimismo, y dado el foco central de análisis, tampoco se analiza un conjunto de otras posibles motivaciones para realizar inversión extranjera en Chile, por ejemplo relacionadas a pago de impuestos o a diversificación de riesgo cambiario y/o riesgo país.

Dentro de la literatura económica, son escasos los trabajos que discuten las motivaciones y efectos de inversión extranjera en la industria del vino. Coelho y de Sousa (2004) describen un conjunto de acuerdos tipo JV en distintos países con producción vitivinícola, argumentando que este tipo de acuerdos se ha convertido, a partir de los años 1980, en uno de los principales instrumentos de internacionalización en la industria de bebidas alcohólicas. En ese trabajo se enfatiza el rol del JV como instrumento para incentivar el intercambio de conocimientos. Así, los acuerdos tipo JV serían motivados por la existencia de distintas habilidades y competencias complementarias de negocio de los socios participantes, cuando esas habilidades

${ }^{6}$ Este trabajo no desarrolla argumentos sobre la comercialización internacional de los vinos exportados. Si bien "acceder a los canales de comercialización internacional de la socia extranjera" constituye una de las motivaciones centrales de viñas locales para participar en un JV con una viña extranjera (The Economist, 1999; Vergara, 2001; Farinelli, 2003), en cerca del $80 \%$ de los JV analizados en este trabajo fue el inversionista extranjero quien impulsó originalmente el acuerdo de JV (ver Anexo 3). De hecho, como se verá, en los JV analizados la comercialización internacional suele quedar a cargo de la socia no chilena. De lo anterior se infiere que el acceso a conocimientos o activos directamente vinculados a la comercialización internacional no son parte de las motivaciones del inversionista extranjero para buscar una socia local en Chile. 
y competencias no pueden ser contratadas directamente mediante una transacción de mercado.

En relación a inversión extranjera en la industria de vinos en Chile, los únicos trabajos que conocemos al respecto son Agosin, Pastén y Vergara (2001), Vergara (2001) y Mac Cawley (2006). Los dos primeros analizan las ventajas ex post logradas por viñas chilenas luego de iniciar un JV con una viña internacional. Ambos estudios son pioneros en recopilar información sobre la existencia de JV vitivinícolas en Chile. En ellos se describe la evolución de inversión extranjera en el sector y los cambios tecnológicos asociados.

En Agosin et al. (2001) se analizan encuestas realizadas a siete viñas chilenas, centrándose en las razones que éstas tendrían para asociarse con una socia extranjera vía un JV. Entre los principales motivos resaltan el lograr acceso a nuevos mercados y a nueva tecnología productiva. Asimismo, se concluye que las viñas locales con acuerdos tipo JV lograron, en los años 1998-1999, mayores precios promedio de exportación en relación a un grupo de control de viñas locales sin JV con alguna socia extranjera.

Mac Cawley (2006), por su parte, analiza los precios por litro exportado, para el periodo 1997-2003, de cuatro JV vitivinícolas con producción en Chile. Este trabajo concluye que las viñas creadas como JV entre una viña local y una socia extranjera presentan un sesgo hacia la producción y exportación de vinos de elevado valor, en relación a los vinos producidos en exclusiva por la correspondiente socia local. El análisis sugiere que para elaborar un vino de alto valor, los JV combinan la tecnología y canales de comercialización de la viña extranjera con los viñedos y conocimientos de la socia local en cuanto a suelo y clima.

Todos los trabajos citados sobre JV vitivinícolas en Chile priorizan el análisis de las motivaciones de, y efectos sobre, la socia local para participar en un acuerdo tipo JV. Por lo mismo, excluyen un análisis explícito de los incentivos de la socia extranjera para participar en un JV. En consecuencia, no analizan la decisión de la socia extranjera para invertir en el JV, en relación a la alternativa de invertir vía una empresa filial. El presente trabajo se concentra en esta última línea de análisis.

El análisis se estructura como sigue. En la sección 2 se describen características económicas relevantes del sector vitivinícola, así como evidencia encontrada respecto a mayores precios de vinos provenientes de JV. La sección 3 revisa literatura teórica sobre la estructura de propiedad óptima de la firma, enfatizando el caso de acuerdos tipo JV. En la sección 4 se plantean hipótesis y argumentos en función de características relevantes para el sector vitivinícola, y luego se presenta la evidencia recopilada al 
respecto. La sección 5 resume las características propias de los vinos de mayor valor unitario que posibilitan la entrada mediante JV. En la sección 6 se concluye.

\section{Hechos estilizados de la industria vitivinícola}

\subsection{Una industria de bienes diferenciados}

La búsqueda de mayor diferenciación de producto incentiva a que en la industria vitivinícola se observen importantes flujos de inversión internacional. La diferenciación de producción vitivinícola entraña múltiples dimensiones. Por un lado, existe diferenciación según la variedad de uva que se utilice para elaborar el vino: uvas tintas en sus distintas variedades - Cabernet Sauvignon, Merlot y otras - y uvas blancas también en distintas variedades (e.g., Sauvignon Blanc o Chardonnay) ${ }^{7}$. Por otro lado, también surge diferenciación en base al concepto de terroir ${ }^{8}$. Según este concepto, un vino tinto del valle del Maipo en Chile (zona central) no tendría las mismas cualidades organolépticas (aroma, cuerpo, aspecto y sabor) que la misma variedad cosechada, por ejemplo, en el Valle de Napa en California. Otra fuente de diferenciación se relaciona con el valor de marca de la viña productora, como también con el valor de marca de vinos específicos. Así, un vino de la viña Baron Philippe de Rothschild ${ }^{9}$ en general no tendrá el mismo valor que un vino de la misma variedad y terroir producido por una

\footnotetext{
${ }^{7}$ Un vino usualmente se produce en base a una variedad de uva. Sin embargo, es frecuente encontrar productos en base a mezclas de dos o más variedades: e.g., Malbec — Syrah, Cabernet Sauvignon- Merlot, entre otros. Una forma alternativa de elaborar un vino es producir un coupage, donde se combinan distintas variedades de uvas, sin que se especifique cuál predomina.

${ }^{8}$ El terroir es un concepto enológico, originado en países europeos con larga tradición de producción vitivinícola, que comprende factores como la temperatura del ambiente, la distribución temporal de las lluvias, horas e intensidad de luz solar, drenaje y otros aspectos que influyen sobre el viñedo y su producción. Según la interpretación 'enológica' de este concepto, distintos terroirs implicarían diferentes cualidades de degustación del vino. Así, la demanda por 'diversidad de terroirs' sería instrumental al producir distintas variedades de vinos (Vendimia, 2002). Sin embargo, el 'terroir' también es interpretable, y probablemente de forma fundamental, como un instrumento de marketing destinado a diferenciar producto según denominación de origen; y junto con lo anterior, también como un instrumento de regulación y protección a grupos específicos de productores regionales (e.g., The Economist, 1998, p. 109).

${ }^{9}$ La viña Baron Philippe de Rothschild pertenece al exclusivo Club des Marques, un grupo de firmas de gran tradición vitivinícola, cuya marca es símbolo de prestigio internacional. Estas firmas vitivinícolas son lo más parecido a las marcas de otras bebidas donde existen denominaciones internacionalmente posicionadas.
} 
viña desconocida. Tal diferenciación se acentúa cuando un vino específico adquiere valor de marca propio. Un ejemplo extremo de esto último lo constituyen los denominados 'vinos trofeo', como es el caso del Chateau Petrus de la viña francesa Moueix, cuyo precio supera los mil dólares por botella (The Economist, 1999).

A nivel mundial, y fundamentalmente desde inicios de los años 1980, se ha ido produciendo un desarrollo creciente de nuevos nichos de mercado para productos vitivinícolas de elevado valor comercial (The Economist, 1999). A su vez, la producción de vinos en estas categorías de valor tiende a usar estrategias productivas y de marketing destinadas a crear percepciones de 'producción escasa' y 'consumo exclusivo' (e.g., Schamel, 2000; The Economist, 2000). Así, las firmas vitivinícolas suelen elaborar sus vinos más caros en escalas reducidas.

El Cuadro $N^{\circ} 1$ presenta una caracterización estándar en la industria, en función de segmentos de mercado definidos según categorías 'precio/ calidad ${ }^{10}$.

CUADRO No 1 CATEGORÍAS SEGÚN PRECIO DEL VINO (MERCADOS INTERNACIONALES) ${ }^{11}$

\begin{tabular}{|c|c|c|}
\hline \multirow[t]{2}{*}{ Segmento de producto } & \multicolumn{2}{|c|}{ Precios FOB de exportación*/ } \\
\hline & $\begin{array}{c}\text { US\$/caja } \\
\text { (12 botellas de } 750 \text { cc) }\end{array}$ & $\begin{array}{l}\text { US\$/botella } \\
\text { (de } 750 \text { cc) }\end{array}$ \\
\hline Ícono & mayor a 50 & mayor a 4,2 \\
\hline Ultra premium & de 45 a 50 & de 3,75 a 4,2 \\
\hline Súper premium & de 35 a 45 & de 2,9 a 3,75 \\
\hline Premium & de 25 a 35 & de 2,1 a 2,9 \\
\hline De mesa & de 18 a 25 & de 1,5 a 2,1 \\
\hline Corrientes & menor a 18 & menor a 1,5 \\
\hline
\end{tabular}

*/ Precios por botella: se han aproximado a partir de valores FOB en US\$ por caja de 12 botellas de 750 cc.

Fuente: Nuevos Mundos: Compendio Vitivinícola de Chile, 2004.

${ }^{10}$ En Chile, los segmentos de mayor importancia en valor exportado son los vino corriente (37\%), de mesa (24\%) y premium (21\%), según datos de la Corporación Chilena del Vino (2005).

${ }^{11}$ Una clasificación similar se encuentra en Mac Cawley (2006), aunque en este caso definida según precios de consumidor final. En este nivel, los vinos íconos alcanzan precios sobre los 50 US\$/botella, entre 14 y 50 US\$/b. los ultra premium, entre 7 y 14 US\$/b. los súper premium, entre 5 y 7 US\$/b. los premium, y bajo 5 US\$/b. el resto. 


\subsection{Uso de JV en segmentos de mercado de mayor valor unitario}

Considerando el caso de la industria chilena, esta sección presenta evidencia sobre el uso más frecuente de JV por parte de inversionistas extranjeros, en relación con la opción de instalar una empresa filial, cuando el objetivo es producir y comercializar vinos de elevado valor comercial.

Durante la década de 1990, Chile se constituyó en uno de los principales destinos de inversión extranjera en Latinoamérica. Durante esa misma década se produce la llegada mayoritaria de inversionistas extranjeros a la industria vitivinícola chilena.

No obstante, la llegada de inversión extranjera a esta industria ya se había iniciado en los años setenta con la creación de una filial de la viña española Miguel Torres. El primer JV se crea en 1988 (viña Los Vascos), negocio conjunto entre la viña francesa Domaines Barons de Rothschild y la viña chilena Santa Rita. Hacia mediados de los noventa, los acuerdos de propiedad compartida llegaban a la decena. En esa década, el flujo total de inversión extranjera en el sector vitivinícola alcanzó en torno a los US\$ 100 millones ${ }^{12}$.

Para reconstruir con mayor detalle cómo se han desarrollado los procesos de inversión extranjera en la industria chilena del vino, se recurrió inicialmente al Compendio Vitivinícola de Chile (Nuevos Mundos, 2004, 2006), en donde se lleva un catastro de viñas y se identifican cuáles corresponden a JV y cuáles a filiales (propiedad $100 \%$ extranjera). Para completar datos faltantes, se analizaron informes de la Corporación Chilena del Vino, publicaciones sobre el sector e información entregada por cada empresa en su página web. Se identificaron cerca de 40 firmas con participación extranjera, incluyendo JV y empresas filiales. Luego de obtener información complementaria a partir de entrevistas en terreno ${ }^{13}$, se obtuvo en definitiva un conjunto consistente de datos para 32 viñas. En el Anexo 1 se presentan las viñas identificadas, sus propietarios y el país de origen de la inversión extranjera.

Una primera aproximación a las disímiles estructuras de especialización productiva observadas, se obtiene al comparar los precios promedio de exportación obtenidos por el grupo de viñas con estructura de propiedad

\footnotetext{
${ }^{12}$ Según datos proporcionados por el Comité de Inversión Extranjera, Gobierno de Chile.

${ }^{13}$ Se realizaron entrevistas a cada una de las empresas identificadas. En la mayoría de los casos se entrevistó personalmente a gerentes de producción, enólogos y gerentes de comercialización de las firmas; un grupo minoritario de viñas respondió vía correo electrónico al cuestionario realizado (véase Anexo 2).
} 
tipo JV versus los precios obtenidos por el grupo de viñas filiales de inversionistas extranjeros. Durante el periodo 1998-2004, el precio promedio de exportación (FOB) obtenido por JV vitivinícolas con producción en Chile fue 4,9 US\$/botella, siendo 2,1 US\$/botella el correspondiente valor para el grupo de viñas filiales. En este mismo periodo, el valor promedio (FOB) del total de vinos exportados desde Chile fue 1,6 US\$/botella (Nuevos Mundos, 2004) $)^{14}$.

Los Gráficos $N^{\circ} 1$ y 2 ilustran el diferente grado de especialización y la distinta importancia relativa de uno y otro tipo de empresas en los distintos segmentos de mercado, definidos según las categorías de precios reportadas en el Cuadro $\mathrm{N}^{\circ} 1$. El Gráfico $\mathrm{N}^{\circ} 1$ informa las participaciones de mercado de cada grupo de empresas en relación con el valor anual total exportado, en cada categoría, por viñas productoras en Chile; en tanto que el Gráfico $N^{\circ} 2$ reporta los porcentajes que representan cada segmento de mercado en los ingresos anuales totales de exportación de uno y otro grupo de empresas (grupos según lo informado en el Anexo 1).

Para los años 2002 y $2004^{15}$ los JV obtienen, en comparación con el grupo de viñas filiales, una mayor proporción del mercado en los segmentos de mayor valor: las viñas JV acumulan una participación entre 23\% y 30\% del valor total exportado de vinos con precios superiores a 4,2 US\$/botella (grupo ícono); mientras que en esa categoría de valor las viñas filiales acumulan entre 8\% y 10\%. En la categoría ‘ultra premium' la participación de mercado de los JV fluctúa entre 9\% y 17\%, mientras que para el grupo de viñas filiales entre $6 \%$ y $12 \%$. Esta diferencia se acentúa en la categoría 'súper premium'. En contraste, en categorías de menor precio (de mesa y corriente), las filiales tienen una participación levemente superior. En definitiva, las viñas tipo JV presentan una mayor participación en los segmentos de producción de vinos de mayor valor.

Una mirada alternativa, que permite corroborar que las viñas tipo JV se especializan con más intensidad en producir vinos de mayor calidad que las viñas filiales, se obtiene a partir de observar las estructuras productivas promedio en cada grupo de firmas. El Gráfico № 2 reporta las participaciones promedio (ponderado) de cada categoría de valor, en los ingresos anuales de exportación de cada grupo de empresas. Las ponderaciones usadas corresponden al valor FOB de las exportaciones de cada firma, respectivamente en los años 2002 y 2004, en relación con el valor anual exportado por

\footnotetext{
${ }^{14}$ Estos precios promedio de exportación se refieren sólo a vinos embotellados; no incluyen vino a granel.

${ }^{15}$ Nada cualitativo cambia si se consideran los valores de las exportaciones anuales promedio realizadas, en otros años recientes, por uno y otro grupo de empresas.
} 
GRÁFICO N ${ }^{\circ}$ 1: PARTICIPACIÓN EN EL VALOR FOB DE EXPORTACIONES, POR SEGMENTO DE PRECIO (EN US\$ POR CAJA) AÑOS 2002 Y 2004
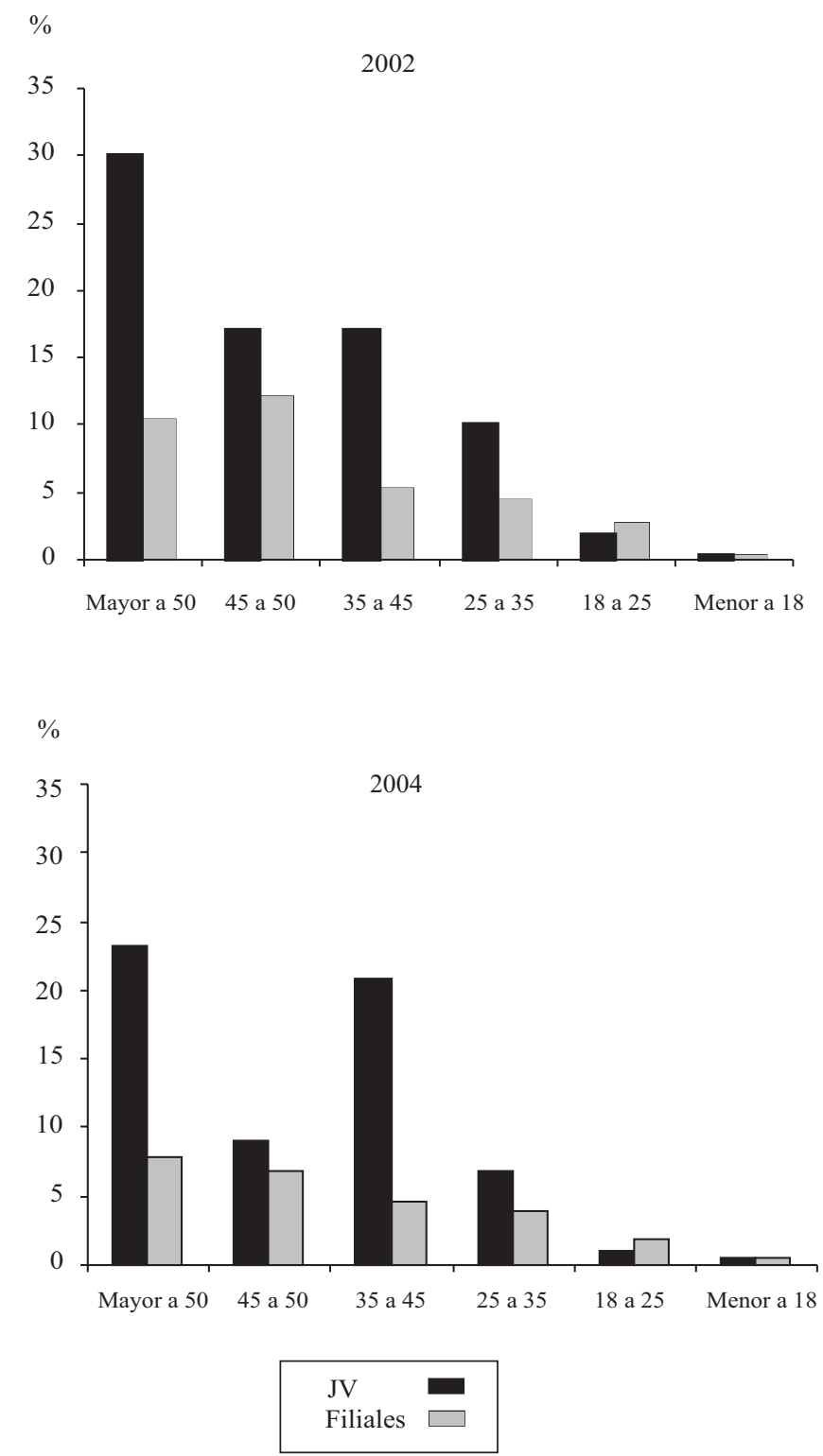

Fuente: Elaboración propia en base a datos de Nuevos Mundos: Compendio Vitivinícola de Chile, 2004, 2006. 


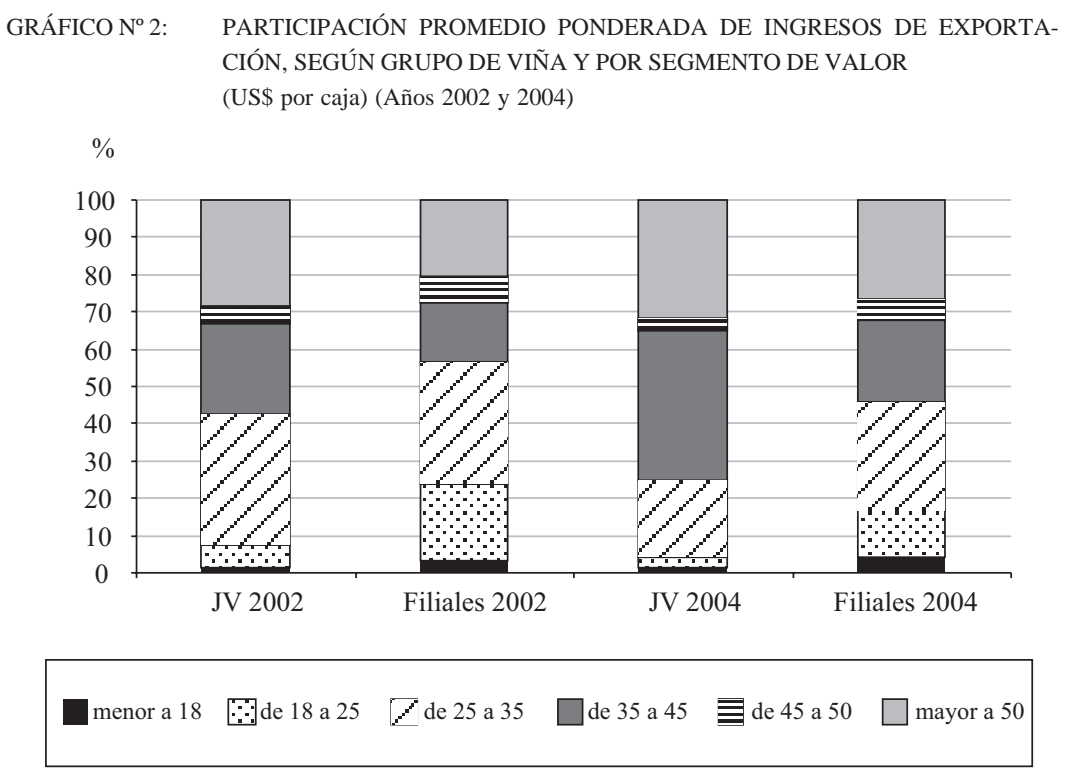

Fuente: Elaboración propia en base a datos de Nuevos Mundos: Compendio Vitivinícola de Chile, 2006.

el total de viñas en cada grupo. Así se toman explícitamente en cuenta los diferentes niveles de ventas de exportación que se observan en el conjunto de viñas consideradas. Se constata que los vinos de mayor precio, en particular las categorías ícono y súper premium, son más relevantes en el caso de los JV que de las filiales.

No obstante lo anterior, este disímil patrón de especialización productiva, de un tipo y otro de viña, podría estar influido por 'efectos escala', en el caso que los tamaños promedio de operación de las viñas incluidas en uno y otro grupo fuesen distintos. Por ejemplo, si las viñas tipo JV fuesen en promedio más grandes que las viñas filiales, y a la vez las viñas más grandes tendieran a especializarse con mayor intensidad que las de viñas más pequeñas en producir vinos más caros, se podría obtener un patrón similar al ilustrado en los Gráficos $N^{\circ} 1$ y 2 . En este caso, la estructura de propiedad podría no ser el único factor que ayude a explicar la disímil especialización productiva, sino también, o incluso exclusivamente, el tamaño de operación de cada viña. El Cuadro $\mathrm{N}^{\circ} 2$ efectivamente ilustra que las viñas JV son en promedio más grandes y que, además, las firmas más grandes se 
CUADRO N ${ }^{\circ}$ 2: $\quad$ PARTICIPACIÓN POR SEGMENTO DE VALOR DEL VINO Y TAMAÑO DE EMPRESA

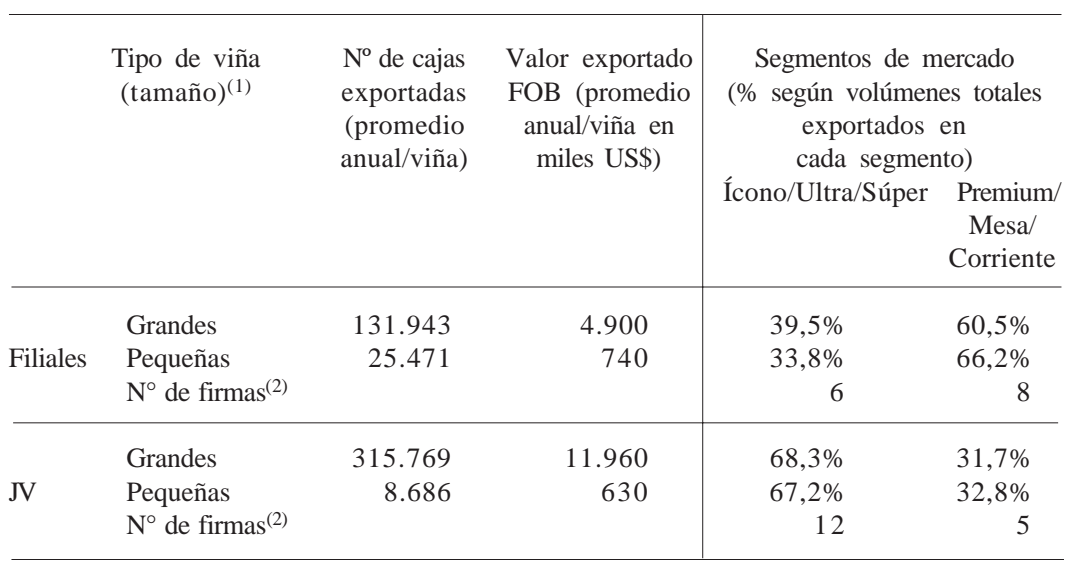

Notas:

(1) Para separar las viñas en grandes y pequeñas se estableció un umbral de 100 mil cajas exportadas en el año 2004. Con esta división se obtienen dos grupos de viñas con manifiestas diferencias en sus respectivas escalas productivas, y ello independiente que se trate de viñas tipo JV o filial.

(2) Corresponde al número de viñas que presentan más del 50\% de sus cajas exportadas en el correspondiente segmento de mercado o categoría de valor.

Fuente: Elaboración propia en base a datos de Nuevos Mundos: Compendio Vitivinícola de Chile, 2006.

tienden a especializar más en vinos de alto valor. Sin embargo, al comparar 'JV grandes' con 'filiales grandes', y 'JV pequeños' con 'filiales pequeñas', se verifica que el patrón relativo de especialización productiva, ilustrado previamente, se mantiene.

\section{3. ¿Por qué propiedad conjunta?}

En los últimos años ha surgido un renovado interés desde la economía por entender los factores que determinan la existencia y estructura de las firmas. Por ejemplo, qué factores determinan que ciertas transacciones se realicen al interior de la firma y otras mediante una institución externa como el mercado.

Como lo señalan Holmstrom y Roberts (1998), entre los factores que determinan la estructura óptima de una firma está la necesidad de resolver diversos problemas de incentivos asociados tanto a asimetrías de informa- 
ción como a dificultades para contratar en forma directa la transferencia de conocimientos ${ }^{16}$.

Respecto al primer punto, la literatura económica se ha concentrado en analizar de qué forma y en qué grado distintas estructuras de propiedad, sobre los activos involucrados en la transacción, brindan incentivos a las partes para realizar las inversiones requeridas por el desarrollo de la firma. Por ejemplo, cuando la inversión no puede ser contratada directamente y, además, es de 'valor específico a la transacción' (en el sentido que la inversión pierde parte o todo su valor si el negocio para el cual se pensó no se realiza ${ }^{17}$ ). Al respecto, en la siguiente subsección se revisa literatura según la cual un arreglo de joint venture puede ser óptimo.

Otros problemas de incentivos que condicionan la estructura de propiedad óptima tienen que ver con la existencia de asimetrías de información que pueden, por ejemplo, impedir brindar una estructura de incentivos apropiada a personal externo a la firma, cuando se requiere que éste cumpla múltiples tareas, o cuando se requieren transferencias de conocimiento y éstas no pueden ser contratadas.

Este último problema ha sido menos estudiado. Al respecto, si bien una proporción significativa de la evidencia indica que estas transferencias se verían facilitadas en organizaciones integradas en una misma firma, también existe evidencia de transferencias exitosas de conocimiento en estructuras de propiedad separadas (e.g., en el caso de proveedores de partes y componentes para el ensamblaje de automóviles en Japón; Holmstrom y Roberts, 1998).

La economía no ha completado aún un cuerpo de teorías del todo consistente en torno a estos temas, excepto sobre el problema de generar incentivos a la inversión (problema conocido en la literatura especializada como hold-up). En la próxima sección se revisa parte de esta literatura; en particular, el modelo básico de Grossman, Hart y Moore y algunas extensiones que permiten entender en qué circunstancias un joint venture podría constituir la estructura societaria óptima.

${ }^{16}$ Otros factores, que no se discuten en el presente artículo, tienen que ver con el uso del mercado como herramienta de monitoreo. Por ejemplo, en organizaciones de tamaño considerable puede ser eficiente separar de la firma algunas unidades de negocios, vendiendo derechos de propiedad sobre ellas y luego usar información de mercado, e.g. cotizando sus acciones en una bolsa de valores, como mecanismo de escrutinio permanente sobre los resultados logrados por esas unidades.

${ }^{17}$ En el caso de la industria del vino, inversión de valor general será toda aquella que diga relación con conocimientos u otros activos cuyo uso y productividad tenga valor similar al elaborar diferentes variedades de vinos y en distintos tipos de terroirs. Inversiones de valor específico se refieren a conocimientos o activos cuyo valor productivo es máximo al aplicarse a esa variedad específica de vino y/o terroir, versus el valor obtenible en otras opciones productivas. 


\subsection{El modelo básico de Grossman, Hart y Moore}

Grossman, Hart y Moore, GHM de aquí en adelante ${ }^{18}$, definen las bases conceptuales para una "nueva teoría de la firma”, siendo su foco los efectos asignativos generados por diferentes estructuras de propiedad, sobre los incentivos para invertir en y cómo usar distintos tipos de activos.

En esta literatura, el concepto de propiedad sobre un activo se entiende como el derecho de 'control residual' sobre tal activo; esto es, el derecho a disponer cómo usar un activo en situaciones no definidas por algún contrato o ley. Así, en circunstancias no contempladas en contratos, el propietario del activo decide unilateralmente sobre su uso.

En esta perspectiva, la estructura de propiedad de un activo se vuelve relevante, desde el punto de vista de la eficiencia asignativa en el uso de ese activo, sólo en un mundo de 'contratos incompletos' ${ }^{\text {'19 }}$. Si fuese posible escribir contratos económicamente completos, tales que describiesen exactamente (i) qué debe hacerse con cada activo en toda posible circunstancia y (ii) cómo repartir los excedentes generados en cada caso, entonces la estructura de propiedad de los activos sería irrelevante desde el punto de vista asignativo. Lo anterior, dado que por contrato podrían especificarse todas las acciones óptimas -i.e. aquellas que maximizan el excedente o creación de valor generado por los usos dados a cada activo-, además de definir, en forma ex ante, cómo dividir el excedente generado entre las distintas partes que estipule cada contrato.

En un mundo de contratos completos un joint venture sería indistinguible de un contrato que especificase, por ejemplo, cómo repartir los beneficios, cómo usar el activo, en qué circunstancias vender y cómo repartir los ingresos de esa venta. En el mundo real, sin embargo, los contratos son incompletos. En este contexto, la nueva teoría de la firma analiza la optimalidad de distintas estructuras de propiedad. La propiedad importa porque,

${ }^{18}$ Grossman y Hart (1986), Hart y Moore (1990).

${ }^{19}$ El concepto de 'contrato incompleto' se define en el sentido que lo hace el enfoque económico, que no necesariamente coincide con el legal. Imagínese la situación de una persona que arrienda mientras espera terminar la construcción de su propiedad. Un contrato que, por ejemplo, especificase que el constructor de la casa debe entregarla en una cierta fecha, que por ello recibirá un determinado pago y que este último se reducirá por cada día de atraso es, desde el punto de vista legal, un contrato completo. Desde el punto de vista económico, sin embargo, si existiesen circunstancias en las que fuese eficiente modificar los términos de este contrato — e.g., si ocurre un terremoto que destruye la construcción un mes antes de la fecha estipulada de entrega - y estas circunstancias no estuviesen consideradas en el contrato originalmente firmado, entonces estaríamos ante un contrato 'económicamente incompleto'. Naturalmente, excepto para transacciones muy simples, la mayoría de los contratos son incompletos desde el punto de vista económico. 
como se mencionó, es el propietario quien decide el uso del activo en caso que surja una situación no especificada en el contrato ${ }^{20}$.

En este contexto, la 'propiedad compartida' de un determinado activo (i.e., un JV en relación al uso de ese activo) significa que las partes en el acuerdo comparten el derecho de control residual sobre ese activo, lo que se traduce — sea de jure o de facto - en un 'derecho a veto', de cada parte, respecto de qué usos son posibles de dar al activo ${ }^{21}$. Así, frente a cualquier circunstancia no especificada en el contrato, las partes que forman el JV deben estar todas de acuerdo para decidir qué uso se dará al activo en cuestión.

Siguiendo esta misma línea argumental, en los próximos párrafos se resumen las ideas centrales de dos modelos relacionados: GHM (Grossman y Hart, 1986; Hart y Moore, 1990) y Cai (2003). El primero ilustra la lógica básica del enfoque de la Nueva Teoría de la Firma y permite entender por qué, bajo los supuestos utilizados por el modelo básico de GHM, una estructura de 'propiedad conjunta' nunca es óptima. En contraste, Cai (2003) plantea un modelo más general al de GHM y deriva circunstancias bajo las cuales 'propiedad conjunta' sí puede ser óptima ${ }^{22,23 .}$

\footnotetext{
${ }^{20}$ Así, por ejemplo, para el caso promedio de una firma pequeña será probablemente su dueño quien decida el uso de los principales activos de la firma. De forma similar, la incompletitud de los contratos también se refleja en una diversidad de relaciones laborales, en las cuales los empleados no tienen preespecificado al detalle qué hacer en cada día, según ocurran tales o cuales eventos, sino que con frecuencia deberán responder a instrucciones que irán cambiando según sean las circunstancias. Esta flexibilidad es deseable en un mundo de 'contratos incompletos'.

${ }^{21}$ Es posible imaginar una diversidad de reglas contractuales que podrían acordarse para definir cómo actuar frente a divergencias de opinión entre las partes en el acuerdo. Sin embargo, el punto general se mantiene: frente a circunstancias no estipuladas vía contrato, la característica de 'propiedad compartida' confiere un derecho de veto a cada parte.

${ }^{22}$ En este enfoque, el carácter 'óptimo’ de una estructura de propiedad está ligado al concepto de eficiencia asignativa. Una estructura de propiedad será óptima si maximiza —en un sentido de 'segundo mejor'- el excedente que genera la relación comercial. Como se explica más adelante, la solución de 'primer mejor' es inalcanzable bajo los supuestos de incompletitud de contratos y especificidad de las inversiones.

${ }^{23}$ Halonen (2001) provee otro marco teórico en el que la propiedad compartida puede ser óptima. El modelo es una versión simplificada del de GHM, pero la interacción comercial se repite infinitas veces. En este modelo, a diferencia de GHM en donde se debe invertir sólo una vez, se asume que la inversión se deprecia en cada periodo y, por lo tanto, debe repetirse. En este contexto, y para ciertas especificaciones paramétricas - relacionadas con la función de costos de la inversión- la propiedad conjunta puede ser óptima e inducir niveles de inversión equivalentes a la solución de primer mejor.
} 
El análisis de GHM parte de un modelo de dos activos $\left(\mathrm{a}_{1} \mathrm{y} \mathrm{a}_{2}\right)$ y dos potenciales dueños/gerentes $\left(M_{1} y M_{2}\right)^{24}$. En este modelo $M_{2}$, utilizando $a_{2}$, proveerá un insumo a $\mathrm{M}_{1}$ quien, utilizando $\mathrm{a}_{1}$, generará un bien final ${ }^{25}$.

Ambos dueños/gerentes pueden, antes de iniciar su relación, realizar inversiones en capital humano, las que les permitirán aumentar el valor del bien final (en el caso de $\mathrm{M}_{1}$ ) o reducir los costos de producción del insumo (en el caso de $\mathrm{M}_{2}$ ). Por ser estas inversiones en capital humano y suponiendo que tales inversiones no son contratables ${ }^{26}$, la presencia en la relación tanto de $\mathrm{M}_{1}$ como de $\mathrm{M}_{2}$ es imprescindible para que puedan obtenerse los beneficios de dichas inversiones. Adicionalmente, estas inversiones se suponen de valor específico a la relación entre $\mathrm{M}_{1} \mathrm{y}_{2}$, en el sentido que su impacto marginal, sobre el excedente creado por la transacción, es máximo cuando $\mathrm{M}_{1}$ y $\mathrm{M}_{2}$ intercambian entre ellas.

El modelo asume que $\mathrm{M}_{2}$ puede vender su insumo a otro comprador para quien el insumo tiene un menor valor (en relación con el precio de demanda de $\mathrm{M}_{1}$ ); mientras que $\mathrm{M}_{1}$ también podría comprar el insumo de algún otro proveedor, aunque este insumo sería menos valioso para $\mathrm{M}_{1}$. Suponiendo un contexto de intercambio en donde la información relevante es completa y simétrica, $\mathrm{M}_{1}$ y $\mathrm{M}_{2}$ siempre intercambiarán entre ellos, dado que ello es eficiente ex post.

En este contexto, GHM analizan la optimalidad de cuatro estructuras alternativas de propiedad: (i) cada gerente es dueño de su activo, (ii) $M_{1}$ es dueño de ambos activos, (iii) $\mathrm{M}_{2}$ es dueño de los dos activos o (iv) ambos gerentes 'comparten la propiedad' de $\mathrm{a}_{1} \mathrm{y} \mathrm{a}_{2}$ (caso equivalente a un JV). Nótese que aun cuando un único gerente sea el dueño de ambos activos, la inversión y participación del otro agente aún es relevante, por cuanto la inversión de la contraparte seguirá determinando el costo del insumo (si se trata de $\mathrm{M}_{2}$ ) o el valor del bien final (si se trata de $\mathrm{M}_{1}$ ).

\footnotetext{
${ }^{24}$ En este modelo, ambos roles se asumen como indistinguibles.

${ }^{25}$ La descripción a presentar corresponde al modelo desarrollado en los capítulos II y III de Hart (1995).

${ }^{26} \mathrm{El}$ supuesto sobre las inversiones es que éstas son 'observables pero no verificables’. Ambos gerentes son capaces de observar cuál es el nivel de inversión propia y del otro gerente, pero una tercera parte (e.g., una corte) no es capaz de hacerlo y por lo tanto es no contratable. Adicionalmente, se asume que el bien a transar (es decir, el insumo que $\mathrm{M}_{2}$ le vende a $\mathrm{M}_{1}$ ) no puede ser descrito ex ante $\mathrm{y}$, por lo tanto, no puede firmarse un contrato que caracterice la transacción que se llevará a cabo en el periodo 1. Llegado el momento, las partes deben negociar un precio y se asume que el resultado de la negociación corresponde a la solución de negociación de Nash. Los pagos que obtendrían los gerentes si no transasen entre ellos (esto es, la outside option de cada una de las partes), junto con el poder relativo de negociación de cada uno, determinan el resultado de la negociación.
} 
En este modelo, y suponiendo que ni uno ni otro gerente tiene $100 \%$ a su favor el poder de negociación, ninguno de los cuatro esquemas de propiedad descritos inducirá niveles de inversión equivalentes a la solución de 'primer mejor', puesto que quien invierte es incapaz de apropiarse completamente del retorno marginal de su inversión. Por ejemplo, un aumento en la inversión de $M_{2}$ reducirá sus costos, sea que $M_{2}$ le venda a $M_{1}$ o a otro agente (aunque la reducción de costos será mayor si le vende a $\mathrm{M}_{1}$ ). Sin embargo, $\mathrm{M}_{2}$ se apropiará sólo de una fracción de la reducción de costos producida por su inversión, esto es, en proporción a su poder de negociación. Como consecuencia, invertirá sólo hasta que su retorno marginal (inferior al retorno marginal 'social') iguale su costo marginal (equivalente al costo marginal 'social'). Así, cualquiera sea la estructura de propiedad, se producirá subinversión en relación a la solución de primer mejor. Y esto, dado que la decisión de inversión es no contratable y de valor específico a la transacción. En este contexto, las diferentes estructuras de propiedad afectarán, en general, el grado de ineficiencia (subinversión) en el nivel de inversión específica que se logre concretar.

Respecto al resto de las estructuras de propiedad consideradas, en el modelo de GHM la 'propiedad compartida' de los activos nunca es 'óptima' (incluso en el sentido de 'segundo mejor'). Esto se debe a que, en el modelo de GHM, los incentivos a invertir provienen de dos fuentes: por un lado, fruto del deseo de aumentar el excedente total, en caso que la transacción se concrete (aunque en este caso quien invierte se apropia sólo de una fracción del mayor excedente creado); y por otro, para mejorar la posición negociadora propia al elevar el pago propio en caso que no ocurra la transacción (i.e., mejorando su outside option). El primer tipo de incentivos para invertir estará presente en todas las estructuras de propiedad analizadas. Sin embargo, la 'propiedad conjunta' de los activos anula completamente el segundo tipo de incentivos, dado el poder de veto que adquiere la contraparte. Así, 'propiedad conjunta' siempre será subóptima, en este contexto de modelamiento, en relación con las otras tres estructuras de propiedad.

Bajo propiedad conjunta de ambos activos ninguna de las partes podrá unilateralmente aprovechar su respectiva outside option, esto es, ninguna de ambas partes podrá, unilateralmente, acordar con un tercero respecto del uso de los activos considerados en el acuerdo de JV. Para llevar a cabo una transacción con un tercero, se deberá tener el consentimiento del socio. En este caso, el socio tendrá incentivos para apropiarse de parte del excedente generado por la inversión de la contraparte. Como resultado, la anticipación de este efecto desincentivará, en algún grado, el nivel de inversión. 
El argumento precedente es válido en el modelo de GHM, en donde cada parte realiza sólo un tipo de inversión (con valor específico a la relación). Generalizando el modelo de GHM, Cai (2003) permite que cada participante en el acuerdo decida sobre dos tipos de inversión: una con valor específico a la relación, y otra de valor general. En términos relativos entre un tipo de inversión y otro, la de valor general impacta marginalmente más sobre el valor de la outside option que sobre el valor dentro de la relación. En este marco, la propiedad conjunta sí puede ser óptima, ya que — siguiendo el argumento del párrafo anterior- será el mecanismo más eficiente para desincentivar la inversión de valor general y, bajo ciertas circunstancias, a la vez ofrecerá los mejores incentivos a la inversión de valor específico.

En el modelo de Cai (2003), los dos tipos de inversión, general y específica, pueden ser sustitutas o complementarias entre sí; siendo este aspecto lo que determina la optimalidad o no de la propiedad conjunta. Los dos tipos de inversión se definen como complementarios si al realizar un tipo de inversión, disminuye el costo marginal del otro tipo de inversión. En el caso de 'perfectos complementos', al aumentar un tipo de inversión es posible aumentar el otro tipo sin costo alguno; en cuyo caso siempre será óptimo aumentar los dos tipos de inversión en forma proporcional. Así, este caso extremo es análogo al contexto de decisión planteado en el modelo de GHM (equivalente a 'como si' existiese sólo un tipo de inversión). Por lo mismo, en este caso extremo la propiedad conjunta nunca es óptima. Así, el modelo de GHM puede pensarse como un caso particular del modelo de Cai (2003).

En contraste, uno y otro tipo de inversión se definen como 'sustitutos perfectos' cuando el costo total de las inversiones sólo depende del valor total (agregado) de ambos tipos de inversión (sin importar cuánto se invierta en cada tipo $)^{27}$. Cuando ambos tipos de inversión son sustitutos (no necesariamente perfectos), la propiedad conjunta resulta ser óptima ya que desincentivará la inversión general e, indirectamente, incentivará la específica. Y esto, por cuanto la propiedad conjunta de los activos evita que inversiones de valor general mejoren la outside option de quien invierte, dado que para hacer uso efectivo de esta opción deberá previamente nego-

${ }^{27}$ Los costos conjuntos de ambos tipos de inversión pueden representarse por la función $C(g, e)=g+e+\gamma(g-e)^{2}$, donde $g$ y $e$ representan las inversiones general y específica respectivamente. Para valores de $\gamma$ bajos los dos tipos de inversiones se vuelven sustitutas ('perfectos sustitutos' en el caso extremo de $\gamma=0$ ). Para valores de $\gamma$ 'suficientemente grandes', siempre se escogerá $e=g$, en cuyo caso uno y otro tipo de inversión serán 'complementarios’. 
ciar con su socio. La inversión específica, por lo tanto, es relativamente más atractiva bajo una estructura de propiedad compartida que bajo un esquema de integración vertical.

\section{Evidencia sobre las teorías presentadas}

Esta sección presenta argumentos específicos de la industria del vino que contribuirían a explicar las disímiles estructuras de propiedad observadas en las inversiones realizadas por inversionistas extranjeros, según la viña se especialice en vinos de mayor o menor valor.

Cuando incentivar inversión es parte de los condicionantes que determinan la estructura óptima de propiedad sobre un determinado activo, la preferencia a favor de propiedad compartida podría explicarse por la necesidad de incentivar inversiones que sean (a) de valor específico y (b) difíciles de contratar en forma ex ante y completa. La concurrencia de las condiciones (a) y (b) genera un problema de hold up, en donde el riesgo de comportamiento oportunista ex post genera un problema de subinversión (Klein et al., 1978). En este contexto, la propiedad compartida puede ser una solución eficiente cuando (i) existe necesidad de realizar, en forma repetida, inversiones de valor específico al negocio en desarrollo, tal como lo sugiere Halonen (2002), o bien (ii) cuanto más fuerte sean los ‘efectos sustitución’ entre inversiones de valor específico al negocio en desarrollo y otras inversiones de valor más general, en el sentido de Cai (2003) ${ }^{28}$.

Por otra parte, asimetrías de información también podrían favorecer la elección de propiedad compartida. Por ejemplo, para incentivar acciones productivas que son difíciles de monitorear, una viña extranjera podría escoger una estructura compartida de propiedad con socios locales, como mecanismo de incentivo de mediano o largo plazo.

En tercer lugar, cuando el conocimiento necesario para producir sea demasiado costoso de adquirir vía compra directa o mediante integración vertical, una firma entrante podría asociarse con otra que ya posea ese conocimiento.

Así, los argumentos a continuación se refieren a desafíos contractuales relacionados con: (a) generar incentivos para desarrollar inversiones de valor específico, (b) superar problemas de agencia asociados a asimetrías de información y (c) promover la transferencia de conocimientos entre las

${ }^{28}$ Dada la definición en Cai (2003) para el concepto de 'inversiones sustitutas', la condición (ii) equivale a exigir que la inversión de valor específico tenga un efecto más acentuado sobre los beneficios netos obtenibles por quien invierte. 
firmas asociadas. El análisis se focaliza en identificar factores que impactan, en las fases de cultivo y elaboración del vino, de manera diferente según sea el valor final del vino producido.

\subsection{Hold-up en viñas}

En la discusión teórica se han enfatizado argumentos relacionados con decisiones de inversión no contratables en forma ex ante y de valor específico de la relación comercial en desarrollo. Sin embargo, las 'inversiones' bajo análisis son interpretables en un sentido más amplio, dado que el problema genérico se asocia con cualquier 'acción' o 'esfuerzo productivo' que posea (i) valor de especificidad y, a la vez, (ii) costos de contratabilidad ex ante. Así, en esta sección el problema central se refiere a problemas de suboferta de alguna acción, esfuerzo productivo o inversión con problemas de contratabilidad y además de valor específico.

Los argumentos a continuación se desarrollan en base a información recopilada vía entrevistas a profesionales del sector ${ }^{29}$ y mediante la revisión de manuales vitivinícolas ${ }^{30}$ y revistas especializadas ${ }^{31}$.

Cuando una viña invierte fuera de su país es probable que enfrente costos informacionales relacionados con aspectos específicos al contexto local de producción. Por ejemplo, cuáles son las tierras más aptas para el cultivo de determinadas variedades de uva, cuál es la exposición óptima a la luz solar y la disponibilidad de agua, cómo combatir las enfermedades locales de la vid, qué nivel de acidez desarrollan las uvas, o con cuáles proveedores conviene contratar. En este contexto, el conocimiento que posean firmas locales será de valor para la firma extranjera.

La firma extranjera deberá entonces definir cuál mecanismo es más eficiente para contratar ese conocimiento local, de forma que el personal contratado use su conocimiento en forma alineada con los objetivos de la firma extranjera. Cuando este conocimiento no sea directamente contratable porque ello es demasiado costoso, la ‘propiedad compartida’ podría llegar a constituir una solución eficiente versus instalar una filial. Así, mientras más relevantes sean los conocimientos locales 'no contratables', mayores debieran ser los costos informacionales asociados con la instalación de una filial.

\footnotetext{
${ }^{29}$ Corporación Chilena del Vino y Oficina de Estudios y Políticas Agrarias (ODEPA)

${ }^{30}$ Corporación Chilena del Vino: Manual de Viticultura (2003)

${ }^{31}$ Revista Vendimia (Corporación Chilena del Vino), Revista del Campo (diario El Mercurio).
} 
Por otro lado, las características particulares de un vino están condicionadas por el terroir donde crecen las vides, en combinación con el clima y las cepas escogidas. Así, una viña elegirá diferentes combinaciones de variedad de uva, tierra, sol, técnicas de riego y poda, según sea su objetivo de producción. Según Coelho y de Sousa (2004), aprender a cultivar una determinada variedad de uva en un nuevo terroir genera costos hundidos durante el proceso de prueba/error y de acumulación gradual de experiencia. En el caso de vinos de más bajo valor comercial, su producción suele requerir inversiones y esfuerzos productivos de valor más estandarizado, dado que se busca producir variedades de uva que se adapten con facilidad a diferentes ambientes y que no requieran cuidados tan específicos.

A continuación se presentan algunos ejemplos sobre esfuerzos productivos, sujetos a costos de contratabilidad, en los cuales se conjetura sobre la existencia de acciones o inversiones productivas que adquirirían mayor valor de especificidad, sea en las fases de cultivo o de elaboración ${ }^{32}$, a medida que aumenta el valor del vino producido. De ser esto cierto, ello contribuiría a explicar el uso más frecuente de 'propiedad compartida’ cuando el objetivo es producir y comercializar vinos de mayor valor comercial $(\text { Cai, 2003) })^{33}$.

(i) Proveedores externos de uva: Mac Cawley (2006) plantea que la calidad final del vino depende muy sensiblemente de las características de la uva cosechada. Para obtener uvas de calidad, son aspectos importantes el restringir el riego ('estrés hídrico') y podar la planta para que los rendimientos por hectárea sean bajos ${ }^{34}$, para así concentrar las cualidades físicas

${ }^{32}$ Es probable que también existan acciones de valor específico y no verificables en la fase de comercialización del vino. Sin embargo, nuestros argumentos se concentran sólo en las fases de cultivo y elaboración del vino, dado nuestro énfasis en analizar las motivaciones del inversionista extranjero al elegir la opción de propiedad compartida. Según datos obtenidos mediante las encuestas realizadas, junto a la revisión de revistas del sector y páginas web de las propias viñas, se infiere que del total de JV considerados en este trabajo, cerca del 75\% de ellos fueron impulsados por la socia extranjera (Anexo 3). Lo anterior, no obstante que "acceder a los canales de distribución internacional de la socia extranjera” sería una de las principales motivaciones de las viñas locales para formar un JV (Mac Cawley, 2006; Agosin et al., 2001; Coelho y de Sousa, 2004).

${ }^{33}$ Toda vez que la empresa inversora enfrente una restricción presupuestaria para realizar inversiones vitivinícolas, mientras más importantes sean las inversiones de valor específico para producir una variedad particular de vino, más importantes tenderán a ser los ‘efectos sustitución’ entre esas inversiones y otras de valor más general (en el sentido de Cai, 2003).

${ }^{34}$ En uva para vino de bajo precio, los rendimientos por hectárea de uva vinífera para un año promedio rondan las 10 toneladas, mientras que en vinos de mayor precio no superan las 4 toneladas en los valles de la zona central de Chile (El Mercurio, 2006b). 
de la uva (Corporación Chilena del Vino, 2003). Por otro lado, y según sean las condiciones ambientales, el tipo de terreno usado y la variedad de uva en crecimiento, se deberá decidir cómo cultivar las vides. En este ámbito una firma extranjera probablemente enfrentará mayores costos de información $^{35}$ al cultivar un nuevo terroir que una firma local. Así, contratar proveedores externos de uva podría ser una decisión relevante para una viña extranjera.

Una viña extranjera podría intentar especificar un contrato de aprovisionamiento de uva con una firma local independiente, indicando el tonelaje requerido, la variedad de uva, el nivel de azúcar y acidez esperada en la uva cosechada, entre otros factores. Sin embargo, esta opción probablemente requerirá que la firma extranjera delegue el control del cumplimiento del contrato en un monitor local, tarea que podría implicar costos de verificación no competitivos para la viña extranjera.

Un ejemplo al respecto se refiere al momento óptimo de cosecha. La Corporación Chilena del Vino (2003) recomienda que el agricultor recorra diariamente la viña cuando se acerca la fecha de madurez, para observar los cambios físicos de la uva, recurriendo incluso al 'mascado' de la piel exterior del fruto, para determinar la fecha óptima de $\operatorname{cosecha}^{36}$. Una firma local probablemente poseerá ventajas informacionales, relativo a una viña extranjera, para verificar el esfuerzo realizado en esta área de decisión, dado su mejor conocimiento del medio local.

Adicionalmente, el conocimiento y manejo de proveedores de uva local con frecuencia será sólo aplicable al país, o incluso región, en donde se encuentran las actividades de cultivo. Así, es probable que este tipo de conocimientos también tenga, en algún grado, valor específico. El uso productivo de estos conocimientos podría lograrse, en principio, vía contrato directo con una viña local independiente. Sin embargo, en este caso podría surgir un problema de subesfuerzo de la contraparte local en la gestión de los proveedores externos, en tanto existan costos de verificación para la viña extranjera.

\footnotetext{
${ }^{35}$ Costos de información respecto de cuán adecuadas pudieran ser determinadas decisiones que requieran el uso de experiencia profesional y que por lo tanto no sean fácilmente verificables.

${ }^{36}$ Según la Corporación Chilena del Vino (2003), para tomar la decisión de inicio de cosecha se utilizan dos medidas para determinar la madurez de la uva: madurez tecnológica y fenológica. La primera se obtiene midiendo el nivel de azúcar y acidez de la uva, mediante un instrumento denominado refractómetro. La segunda, complementaria a la primera, se basa en la observación de los frutos y la acumulación de taninos en la piel del mismo, lo cual es función de las condiciones del terreno. Esto último no es cuantificable.
} 
Asimismo, el manejo de proveedores de uva tiende a adquirir mayor importancia relativa al producir vinos de mayor valor. Estos últimos suelen requerir mayores cuidados al cultivar la uva; entre otros, para mantener rendimientos por hectárea que aumenten la concentración de las propiedades organolépticas de la uva, o para minimizar el daño ocasionado a los racimos cosechados, o para adaptar tareas específicas de cultivo a la variedad particular de uva en producción. En contraste, en vinos de menor valor se tiende a utilizar uvas de calidad estándar, perdiendo importancia relativa el manejo de los proveedores de uva.

(ii) Contratación de temporeros: Al cultivar uvas para producir vinos de elevado valor, con frecuencia se utiliza cosecha manual (Troncoso et al., 2002). Para ello se contratan trabajadores de temporada, los cuales son capacitados por las propias viñas en relación con los cuidados requeridos en la recolección de la uva (El Mercurio, 2006a).

La cosecha manual implica costos de entrenamiento, monitoreo y verificación del esfuerzo productivo de los temporeros contratados. Es posible que una firma local tenga ventajas informacionales - respecto a una firma extranjera - para verificar la capacidad y el esfuerzo de esta fuerza laboral.

Las técnicas de cosecha manual en general adquieren mayor importancia al elaborar vinos más caros, por cuanto en estos segmentos de valor el precio final del vino estaría más fuertemente condicionado, en forma inversa, por daños físicos ocurridos a la uva durante la cosecha (Troncoso et al., 2002). Por otro lado, invertir en tecnología mecanizada de cosecha involucra inversiones de valor más general, en comparación con la cosecha manual (con mayor valor de especificidad según el tipo de uva por cosechar), considerando que la tecnología mecanizada puede ser utilizada en una amplia diversidad de tipos de frutos (no sólo uva).

(iii) Experiencia del enólogo: Otro ejemplo se refiere a tareas requeridas en la fase de elaboración del vino. Al momento que los vinos fermentan, se les suele dejar que se 'oxigenen' (Giuliani, 2002) ${ }^{37}$. El momento para iniciar tal oxigenación se conoce a partir de un aroma particular, cuya identificación es responsabilidad del enólogo y en donde su experiencia importa. Esta decisión es especialmente relevante en la elaboración de vinos de ma-

${ }^{37}$ El proceso de oxigenación se utiliza en vinos con crianza en barrica, o en aquellas variantes jóvenes que, por su estructura, logren mediante la oxigenación, o la decantación, despertar su potencial aromático. Esta oxigenación cataliza los cambios fisioquímicos que se producen en el vino; en definitiva, su maduración. 
yor valor, por cuanto se busca evitar que el vino reciba demasiado oxígeno y con ello se oxide. En la medida que una firma local enfrente menores costos informacionales para contratar los servicios relevantes de enología (e.g., porque, como ocurre a veces, el socio local es el enólogo o porque la firma local tiene un enólogo con experiencia local), mayor será la conveniencia de realizar un joint venture cuando se planee producir vinos de mayor valor.

La tarea del enólogo consiste en procesar la uva de forma de maximizar su valor de mercado. Dado que el vino elaborado tendrá diferentes características según sea la combinación particular de variedad de uva y tierra de cultivo, es probable que exista algún valor de especificidad en los conocimientos desarrollados por el enólogo respecto de variedades particulares de vino en elaboración.

Por otro lado, a mayor valor del vino la experiencia del enólogo tiende a adquirir mayor importancia. Por ejemplo, para producir vinos de elevado valor el enólogo debe saber cuál es el momento de maduración del vino y qué composición química se puede lograr a partir de los mostos de que dispone. Al elaborar vinos de menor valor, la experiencia del enólogo pierde importancia relativa.

Los ejemplos previos apoyan la hipótesis de que 'propiedad compartida' podría constituir una estructura de propiedad eficiente para incentivar determinadas inversiones y/o acciones con valor específico y no contratables (directamente) ${ }^{38}$. Asimismo, los argumentos expuestos sugieren que este tipo de inversiones y/o acciones tienden a adquirir mayor relevancia a medida que aumenta el valor del vino en producción.

A continuación se presentan argumentos sobre problemas de información asimétrica y transferencias de conocimiento, desarrollados a partir de evidencia obtenida en las encuestas realizadas a viñas.

\subsection{Asimetrías de información}

Junto a la no contratabilidad de acciones o inversiones de valor específico, es probable que la viña extranjera también enfrente información asimétrica respecto de otras tareas realizadas por personal local, debiendo

${ }^{38}$ Existen acciones productivas de valor específico que no necesariamente son 'no contratables'. Por ejemplo, en el cultivo de uva para vinos de menor valor la técnica de poda suele involucrar esfuerzos con un alto grado de estandarización: el objetivo predominante suele consistir en maximizar los rendimientos de kilos cosechados por hectárea. En contraste, en la producción de vinos de mayor valor las técnicas de poda presentan mayor heterogeneidad y valor de especificidad. No obstante, la técnica de poda es una variable con relativamente bajos costos de verificación. 
asumir costos de verificación al contratarlas. Al respecto, la estructura de propiedad óptima de la firma debiera asignar los derechos de control, sobre las ganancias residuales del negocio, a aquella parte en la transacción que enfrente los menores costos informacionales para contratar los esfuerzos requeridos. De esta forma se favorece la ejecución de acciones costosas de verificar y/o la revelación de características que sean información privada de alguna de las partes involucradas.

A continuación se presentan ejemplos de problemas de información asimétrica que debieran ser más costosos de resolver, en general, para la filial de una viña extranjera que para una socia local. Asimismo, se argumenta que la relevancia productiva de estas asimetrías de información tenderá a aumentar mientras más alto sea el valor del vino en producción.

Como principio general, es probable que una empresa filial enfrente mayores costos para monitorear el esfuerzo productivo del personal local; como también mayores costos para averiguar si el personal local posee o no las habilidades requeridas por la producción objetivo.

$\mathrm{Al}$ respecto, pueden existir asimetrías de información asociadas a problemas de selección adversa. Por ejemplo, que la firma extranjera no sepa si el personal local tiene el conocimiento suficiente sobre las tareas de cultivo, elaboración y/o administración que se necesitan para producir una determinada variedad de vino. O cuán adecuado es el terroir escogido para producir esa variedad. En ambos casos, una socia local probablemente enfrentará menores costos para verificar estos atributos. Asimismo, parece razonable presumir que la importancia de estos costos de verificación aumentará mientras más alto sea el valor de la producción objetivo.

Piénsese ahora en la contratación de temporeros durante la época de cosecha. En este periodo se suelen generar excesos de demanda por mano de obra capacitada para esta tarea. De hecho, es frecuente que los buenos cosechadores de una viña sean luego recontratados por otra viña, tentados por un pago mayor. Ello provoca fuerte competencia entre las viñas (El Mercurio, 2006a). En este contexto, es probable que las negociaciones con contratistas, que representan y agrupan a los temporeros, sean más costosas de resolver para una firma extranjera. Así, el inversionista extranjero deberá contratar un administrador local para negociar con los contratistas. Dado que la firma extranjera conoce menos el contexto local, probablemente le será más costoso que a una socia local monitorear al administrador local.

En cuanto a tareas de aprovisionamiento externo de uva, es posible que también existan asimetrías de información, respecto de la calidad de las uvas, que podrían ser resueltas en forma eficiente por un acuerdo tipo JV. El 
cuestionario aplicado a las viñas incluyó una pregunta sobre el uso de contratos de provisión externa de uva (Anexo 2). En Chile se usan fundamentalmente dos mecanismos para contratar la provisión de uva con otras viñas $^{39}$. Por un lado, contratos de largo plazo entre viñas. Por otro, intermediarios compran uva a viñas productoras y luego la venden a elaboradoras de vino. Según la evidencia recabada en las entrevistas, son escasas las viñas que se autoabastecen por completo de uva ${ }^{40}$.

Los resultados de la encuesta indican que las viñas organizadas como JV tienden a comprar con más frecuencia, en comparación con las viñas filiales, uva producida por terceros: $64 \%$ del total de JV encuestados utilizan contratos de provisión externa de uva versus $43 \%$ en el grupo de viñas filiales.

En el grupo de viñas filiales que utilizan proveedores externos de uva destacan un par de casos. En ambos la viña extranjera ha logrado acumular conocimiento sobre el contexto local. En el primer caso, la viña Baron de Rothschild tiene, a partir del año 2000, una filial del mismo nombre en Chile y a la vez participa en el JV Almaviva junto con Concha y Toro (desde 1996). Apoyada por su participación en Almaviva, la filial chilena de Baron de Rothschild es probable que haya logrado acceso a conocimiento local que no necesariamente tienen otras filiales de viñas extranjeras. En el segundo caso, la viña Miguel Torres se encuentra en Chile desde mediados de los años 1970, siendo la filial más antigua de una viña extranjera con operaciones en Chile. Por lo anterior, sería de esperar que haya acumulado conocimiento sobre el manejo de proveedores locales de uva.

Las viñas con JV que no utilizan proveedores externos de uva tienden a ser firmas en las que una parte significativa de su negocio consiste en vender uva a otras viñas. Un ejemplo al respecto es la viña Terravid.

\subsection{Uso e intercambio de conocimiento no contratable}

Ajustes en la estructura de propiedad sobre activos productivos también pueden actuar como un mecanismo eficiente para incentivar el uso o intercambio de conocimientos no directamente contratables vía una tran(ODEPA)

${ }^{39}$ Según consultas realizadas en la Oficina de Estudios y Políticas Agrarias

${ }^{40}$ Según datos de la Corporación Chilena del Vino, las viñas que producen y exportan vinos desde Chile compran cerca del $70 \%$ de su demanda total de uva a pequeños y medianos productores independientes. Datos del Servicio Agrícola y Ganadero de Chile (SAG) señalan que, en el año 2005, de las 13.844 propiedades catastradas con parronales de uvas para vinificación en Chile, más de 10.000 no superaban las 5 hectáreas (El Mercurio, 11/03/07). 
sacción de mercado. En esta sección se argumenta que, en caso de enfrentarse costos muy elevados para verificar el uso o traspaso de un determinado conocimiento o experiencia laboral, el compartir derechos de propiedad, vía un acuerdo tipo JV, puede favorecer el uso o transferencia de conocimientos entre las firmas socias, más que la integración vertical que implica una filial.

A continuación se presenta evidencia obtenida en las encuestas realizadas a viñas con participación de inversionistas extranjeros (incluyendo JV y filiales). Entre otros temas (ver Anexo 2), se le consultó a cada viña sobre el país de origen del personal que trabaja en las siguientes áreas: cultivo de la uva, elaboración en bodega, administración y comercialización. También se les consultó sobre cuáles áreas de actividad estaban a cargo de cada socia.

Respecto al manejo de esfuerzos en la fase de cultivo, sólo 8\% del total de viñas encuestadas respondió que en esta área trabajan profesionales extranjeros. Esta evidencia es consistente con la hipótesis de que el conocimiento local sobre el manejo de cepas, la tierra y el clima, entre otros factores, es de valor para el inversionista extranjero"41.

En relación al área de administración y gerencia general, 45\% del total de filiales encuestadas respondió que sí contrataban profesionales extranjeros en esta área. En el grupo de las viñas con JV, sólo el 10\% respondió afirmativamente. Según las respuestas recibidas, en los acuerdos tipo JV la socia local usualmente queda a cargo de la administración de la viña, dado que es ella quien mejor conoce los distintos aspectos legales de contratación con proveedores y, en general, de contratos con personal local.

El área de comercialización, en cambio, es dirigida por profesionales extranjeros en un $80 \%$ del total de casos encuestados (filiales y JV). Esto es consistente con que la socia o matriz extranjera quede mayoritariamente a cargo de la comercialización internacional del producto. Según las encuestas realizadas, los JV vitivinícolas en Chile tienen en común que sus ventas en mercados internacionales se realizan mayoritariamente vía los canales de

${ }^{41}$ A modo de ilustración al respecto, existen enfermedades de las vides que tienen una incidencia particular en Chile. Una de éstas es el oidio, que se da en climas secos y por lo tanto no es frecuente en países de Europa central. Su tratamiento requiere calendarizar los días de aplicación de químicos, lo cual varía según la zona. A ello se suma la existencia de insectos locales que afectan a las vides. Por otra parte, el clima seco de Chile requiere que exista riego, lo que no ocurre en Europa (según entrevistas en la Corporación Chilena del Vino). Finalmente, la alta productividad de las viñas en Chile, respecto de las europeas, se da al existir diferencias entre las curvas de radiación solar de ambas zonas. 
distribución de la socia extranjera. Por ejemplo, el vino Almaviva (JV entre Concha y Toro y la viña francesa Baron de Rothschild) es de los pocos vinos 'no franceses' que son comercializados por négociants en los mercados internacionales ${ }^{42}$ (El Mercurio, 2005).

Por lo tanto, las viñas locales aportarían fundamentalmente sus conocimientos sobre cultivo, enología y administración. Mientras que las viñas extranjeras aportarían sobre todo sus conocimientos en enología y canales de comercialización internacional.

Ahora bien, ¿por qué los conocimientos de una y otra socia no serían directamente contratables en el mercado, debiéndose recurrir a compartir el control residual sobre las rentas del negocio?

Históricamente la elaboración de vinos ha dependido en forma significativa de "la destreza manual y percepción sensorial de especialistas" (Giuliani, 2002). Esto ha determinado estructuras productivas fuertemente basadas en la experiencia y acumulación de conocimiento tácito ${ }^{43}$. En años más recientes el conocimiento vitivinícola se ha vuelto más codificable, especialmente respecto de los principios químicos y biológicos que subyacen en la elaboración de vinos (Unwin, 1991). No obstante, el conocimiento tácito continúa siendo un componente importante de la experiencia acumulada por el experto agronómico y el enólogo.

En su análisis sobre el cluster del vino en el valle chileno de Colchagua, Giuliani y Bell (2005) argumentan que en este sector no se observan flujos de información ‘ $100 \%$ codificables', respecto de cómo producir y comercializar los vinos. Plantean que el aprendizaje y difusión de conocimiento vitivinícola se tiende a producir en círculos limitados, con integrantes que pertenecen a una misma comunidad y que suelen compartir un código propio de comunicación.

A modo de principio general, mientras más relevante sea el acceso a conocimientos no codificables, mayor debiera ser el interés de un nuevo entrante para asociarse con quien tenga acceso a tales conocimientos (una viña local). Por otro lado, parece razonable presumir que la relevancia productiva de este tipo de conocimiento tenderá a aumentar conforme se trate de vinos de mayor valor.

${ }^{42}$ Los négociants son comercializadores especializados en vinos franceses de elevado valor. Negocian el precio y colocan estos vinos en los mercados internacionales, estando entre sus clientes desde exclusivos hoteles y restaurantes, hasta centros especializados de distribución minorista.

${ }^{43} \mathrm{El}$ conocimiento tácito puede definirse como aquel cuyo traspaso, entre distintos agentes económicos, involucra costos muy elevados. Fruto de ello, tal conocimiento no será contratable vía una transacción directa de mercado (Polanyi, 1967). 
En las encuestas realizadas a viñas chilenas se obtuvo evidencia de que efectivamente ocurren transferencias de conocimiento. La transferencia de conocimientos se suele dar a partir de 'asesorías mixtas', en las cuales el profesional extranjero visita Chile durante la temporada de cosecha y de elaboración del vino, mientras que los agrónomos y enólogos chilenos visitan el país de origen de la socia o matriz extranjera en la segunda mitad del año, cuando comienza la cosecha y producción en el hemisferio norte.

En relación a la fase de cultivo, en ninguno de los dos tipos de estructura de propiedad se encontró evidencia de intercambios significativos de profesionales: sólo un 15\% de los JV encuestados dijo utilizar asesorías mixtas, mientras que ninguna filial lo hacía.

En el área enológica, en cambio, se encontró evidencia de intercambio relevante de conocimiento entre profesionales locales y extranjeros, sobre todo en las viñas con acuerdos JV. En el 70\% del total encuestado de JV existían asesorías permanentes de enólogos extranjeros. En las filiales, 58\% del total encuestado utiliza personal extranjero permanente y sólo un $17 \%$ asesorías entre personal de la filial y de la viña matriz.

Los resultados anteriores aportan indicios de que en acuerdos tipo JV se tiende a producir mayor transferencia de conocimientos, sobre todo en relación a decisiones enológicas en la fase de bodega, respecto de las inversiones bajo integración vertical (filiales).

En un JV tanto la firma local como la extranjera tendrían interés en ampliar los conocimientos de su personal a partir de asesorías mixtas. Bajo integración vertical, en cambio, la viña extranjera podría privilegiar que sea su personal extranjero el que acumule mayor conocimiento. En este caso, los enólogos locales tendrían menos incentivos a transferir conocimiento propio a la firma extranjera.

\subsection{Otras hipótesis}

Esta sección analiza dos hipótesis adicionales que también podrían contribuir a explicar, en principio, el uso más frecuente de JV, por parte de inversionistas extranjeros, en la producción chilena de vinos de mayor valor. No obstante, en definitiva se descarta la relevancia de estas hipótesis.

(i) ¿Utilizar un JV como instrumento para acceder al mercado nacional?

Como ya se ha argumentado, una fuente de motivación de las viñas internacionales para invertir vía un JV con un socio local es lograr un acceso ventajoso a activos valiosos para la producción y/o comercialización de 
la producción objetivo. Al respecto, es directamente descartable que un objetivo central de la inversión extranjera sea acceder al mercado chileno a partir de una marca (viña) local ya establecida. Tanto los JV como las empresas filiales en Chile exportan en promedio más del 90\% de su producción (Nuevos Mundos, 2004).

No obstante lo anterior, al asociarse con una viña chilena el inversionista extranjero podría quizás buscar complementar sus actuales canales de comercialización. Sin embargo, los JV en Chile realizan la comercialización internacional de su producción usando, fundamentalmente, los canales de la socia extranjera. Relacionado con esto, un $40 \%$ del valor total exportado por viñas tipo JV tiene como destino al país de origen de la socia extranjera (Corporación Chilena del Vino, 2005).

\section{(ii) ¿Usar un JV para lograr acceso a un nuevo terroir?}

Otra posible motivación para preferir un JV podría tener relación con restricciones para acceder a algún terroir específico; por ejemplo, en caso que existan limitaciones para comprar la viña local que posee el terroir demandado o para comprar directamente el área de interés.

Dado que en Chile no existen restricciones legales específicas a la compra de tierras, esta posibilidad sólo podría darse si viñas locales no estuviesen dispuestas a vender las áreas en demanda. Así, sólo inversionistas que lograsen asociarse con firmas locales tendrían acceso a los terroirs buscados. En este caso sería esperable un disímil patrón de ubicaciones entre las viñas de agentes extranjeros que invierten bajo la modalidad de JV versus inversiones realizadas bajo la modalidad de empresa filial (logrando los JV acceso, en promedio, a mejores áreas de cultivo).

Los valles vitivinícolas más importantes en Chile han correspondido históricamente a regiones centrales, entre éstas los valles de Maipo, Rapel, Curicó y Maule, incluyendo las zonas de Pirque, Buin, Cachapoal, Colchagua y Talca (Del Pozo, 1998). En este grupo se debería también incluir el valle de Casablanca, que en años recientes ha pasado a ser el más importante valle productor de uvas blancas (Anexo 4). Sin embargo, actualmente nuevas viñas se instalan cada vez más fuera de las regiones tradicionales, en busca de nuevos terroirs ${ }^{44}$. Así, los valles de Limarí, Itata y Aconcagua son hoy zonas vitivinícolas en expansión (La Segunda, 2005).

\footnotetext{
${ }^{44}$ Por ejemplo, Concha y Toro adquirió la viña Francisco de Aguirre en el valle de Limarí durante el 2005. Y desde 1998, la Viña Momberg es la viña más austral del país, situándose en la XI Región (más allá del paralelo 43).
} 
El Cuadro $\mathrm{N}^{0} 3$ presenta la distribución de hectáreas vitivinícolas y el número de viñas existentes, en los principales valles vitivinícolas de Chile, considerando el total de viñas con capital extranjero que han sido incluidas en el presente estudio (ver Anexo 1$)^{45}$.

La localización de las viñas con JV se concentra en los valles centrales, con el 97\% del total de sus hectáreas vitivinícolas en los valles de Maule, Maipo, Rapel (conformado por los valles Cachapoal y Colchagua) y Casablanca. Las viñas filiales presentan una distribución algo más dispersa: el 69\% del total de sus hectáreas vitivinícolas se distribuyen en los valles de Maule, Maipo, Rapel y Casablanca, aunque también poseen zonas de cultivo en donde no existe inversión vía JV (e.g., en el valle del río Itata).

A partir de las entrevistas realizadas no se encontró evidencia concluyente sobre la relevancia de la hipótesis de 'acceso restringido a un determinado terroir'. Algunas viñas aseguraron que, al formar un JV, las firmas asociadas realizaron esfuerzos conjuntos por encontrar un nuevo terroir $^{46}$. Considerando el total de las viñas encuestadas con acuerdos de

CUADRO No 3: HECTÁREAS CULTIVADAS*/Y NÚMERO DE VIÑAS

(SEGÚN SISTEMA CONTRACTUAL DE INVERSIÓN, AÑO 2004)

\begin{tabular}{lrrrrrrr}
\hline \multirow{2}{*}{ Valles } & \multicolumn{3}{c}{ Hectáreas cultivadas } & & \multicolumn{2}{c}{ Cantidad de viñas } \\
\cline { 2 - 4 } & JV & $\%$ & Filiales & $\%$ & & JV & Filiales \\
\hline Maipo & 325 & $16,0 \%$ & 233 & $16,2 \%$ & & 6 & 4 \\
Maule & 283 & $13,9 \%$ & 186 & $12,9 \%$ & & 4 & 2 \\
Rapel & 950 & $46,7 \%$ & 390 & $27,1 \%$ & & 4 & 3 \\
Casablanca & 411 & $20,2 \%$ & 180 & $12,5 \%$ & & 4 & 3 \\
Aconcagua & 36 & $1,8 \%$ & 25 & $1,7 \%$ & & 2 & 1 \\
Itata & 0 & $0,0 \%$ & 34 & $2,4 \%$ & & 0 & 1 \\
Curicó & 30 & $1,5 \%$ & 392 & $27,2 \%$ & & 1 & 2 \\
Total & 2.035 & & 1.440 & & 21 & 16 \\
\hline
\end{tabular}

*/: Hectáreas cultivadas por viñas que poseen tierras en cada valle, siguiendo la clasificación del Decreto Nº 464 del año 1995.

Fuente: Elaboración propia en base a datos de Nuevos Mundos: Compendio Vitivinícola de Chile (2004, 2006) y Corporación Chilena del Vino (2005).

${ }^{45}$ Existen casos en los que empresas vitivinícolas tienen viñas en más de una localización. El Cuadro $N^{\circ} 3$ considera todas las áreas que posee cada firma. Aunque las firmas con múltiples localizaciones son minoritarias (corresponden a las viñas locales más grandes).

${ }^{46}$ Por ejemplo, Francois Walewski, gerente general de viña Altair, afirma que estuvieron 6 meses buscando un terroir apropiado para el JV. En el caso de la viña Seña (JV entre Viña Errázuriz y Robert Mondavi) tardaron 4 años en encontrar el terroir adecuado. 
JV, en la mitad de ellas se realizaron esfuerzos conjuntos de búsqueda para adquirir nuevas áreas de producción, como alternativa al uso de tierras ya en propiedad de la socia local.

En definitiva, y dada la evidencia recolectada, la hipótesis de acceso restringido a algún terroir no surge como explicación del todo convincente sobre las motivaciones para preferir acuerdos de JV. Sin embargo, la evidencia citada tampoco descarta que inversionistas extranjeros perciban a un JV con una viña local como un mecanismo que facilita el esfuerzo de búsqueda de un nuevo terroir.

La hipótesis de 'acceso restringido a un determinado terroir' podría incluir una dimensión adicional. Morton y Podolny (1998) analizaron un conjunto de viñas en California, encontrando que los propietarios de algunas viñas tenían motivaciones 'no estrictamente financieras' en su lógica de decisiones. Algunos de estos propietarios obtenían 'utilidad' por el simple hecho de ser ellos, o algún familiar directo, los que administraban el negocio.

Extrapolando este argumento al caso chileno, podría plantearse la hipótesis que viñas locales no quieran vender sus propiedades vitivinícolas, aun cuando la oferta de compra fuese 'financieramente rentable'. Esto ayudaría a explicar la existencia de viñas con propiedad conjunta. Acuerdos tipo JV serían el instrumento mediante el cual firmas extranjeras participan en viñas locales que son propietarias de terroirs de interés.

Sin embargo, y en directa contradicción con la hipótesis previa, entre un $30-40 \%$ del total de las filiales encuestadas nacieron a partir de la adquisición de viñas locales. Mientras que más de un tercio de los JV, de los creados hasta el año 2002, se generaron a partir de la unión con empresarios locales que a esa fecha no poseían establecimientos vitivinícolas (Nuevos Mundos, 2004).

\section{Vinos ícono y ultra premium}

Hasta este punto el análisis se ha realizado sobre la base de diferenciar en términos genéricos entre vinos de mayor y menor precio. $\mathrm{Al}$ respecto, cabe resumir un conjunto de aspectos que implican diferencias de fondo entre las distintas categorías de vinos (ícono, premium, corrientes, etc.).

Según expertos del sector ${ }^{47}$, los vinos ícono y ultra premium presentan ciertas características que los diferencian claramente de vinos de menor

${ }^{47}$ Por ejemplo, véase www.chilevinos.cl: "El Dilema de los Íconos”, 13 de enero de 2006. 
valor (e.g., vinos premium o corrientes). En primer lugar, el primer tipo de vinos posee una alta calidad, identificable y repetible en el tiempo. Tal calidad es 'certificada' regularmente en competencias internacionales y en revistas especializadas. En este tipo de vinos, el proceso productivo, tanto de la uva como del vino propiamente tal, adquiere particular relevancia en términos de condicionar el valor comercial del producto final. Así, por ejemplo, la decisión respecto al momento de cosecha y la supervisión del proceso manual de cosecha son condicionantes especialmente relevantes al elaborar vinos de mayor valor. Y como ya se argumentó previamente, estructuras de propiedad compartida reducirían los costos contractuales para un inversionista extranjero que busca desarrollar este tipo de producción.

En segundo lugar, el viñedo productor o terroir de un vino ícono o ultra premium debe tener determinadas particularidades, sea por ejemplo según la edad de las vides, características del suelo, o exposición a la luz solar. Tales especificidades facilitan la diferenciación del producto final. Al respecto, la selección del terroir típicamente requiere de conocimientos locales de difícil (costosa) contratación.

En tercer lugar, expertos sectoriales señalan que esfuerzos de marketing y venta también juegan un rol significativo, y claramente más importante que en el caso de vinos de menor valor, al momento de comercializar vinos de mayor valor. Los vinos ícono, ultra premium y súper premium normalmente se comercializan en tiendas especializadas, gastronomía de elite y clubes de degustación de vino, para lo cual se recurre a distribuidores directos, exclusivos y especializados (Nuevos Mundos, 2004). Generalmente, en un joint venture es el socio extranjero quien tiene acceso a estas redes especializadas de distribución. Los segmentos de precios menores, en contraste, se comercializan principalmente en supermercados.

En síntesis, debido a las características distintivas mencionadas para el caso de vinos de mayor valor, diferentes acciones realizadas en los procesos de cultivo, cosecha y fermentación del vino juegan un rol relevante en la estrategia de diferenciar el producto final. Y esto, tanto en términos de las características propias del vino producido como para efectos de potenciar su comercialización final. En definitiva, y en función de cuál sea la fuente relevante de diferenciación del producto, un vino de mayor valor requiere realizar distintas inversiones y acciones, las que, según la tesis analizada en este trabajo, serían más eficientes de contratar e implementar bajo esquemas de propiedad compartida. 


\section{Conclusiones}

Este trabajo ha analizado la decisión de inversionistas extranjeros para realizar inversiones vitivinícolas en otro país vía 'acuerdos de propiedad compartida' (joint venture) con un socio local, o bien invirtiendo mediante una empresa filial. La evidencia revisada, sobre la industria vitivinícola en Chile, indica que acuerdos tipo JV son usados con mayor frecuencia cuando el objetivo del inversionista coincide con una mayor especialización en la producción y comercialización de vinos de elevado valor comercial.

En la industria del vino, al igual como ocurre en otras industrias de alimentos y de licores (tipo 'spirits') en las cuales existe fuerte diferenciación de producto y en donde los valores de marca y 'denominaciones de origen’ agregan valor al producto, como por ejemplo ocurre con las industrias del whisky, de quesos y de jamones curados (ibérico, prosciutto y otros similares), es frecuente que los esfuerzos de diferenciación del producto no sólo descansen en factores de tradición y ambientales sino también en una "fanática atención al detalle", según lo describe The Economist $(2006)^{48}$.

Consistente con esta descripción informal de industrias de alimentos y licores con fuerte diferenciación de producto, la hipótesis central discutida en este trabajo es que a medida que aumentan los esfuerzos para diferenciar el vino y comercializar productos de elevado valor comercial, ello tiende a requerir inversiones, intercambios de conocimiento y un conjunto de otros esfuerzos productivos que poseen valor específico al nuevo producto, y respecto de los cuales su contratación directa sería con frecuencia, además, muy costosa. Tales inversiones, conocimientos y esfuerzos de valor específico generarían efectos más significativos, sobre el valor final del producto, a medida que se intenta vender en segmentos de mercado con cada vez mayor valor unitario.

La evidencia teórica y empírica revisada sugiere que, frente al desafío de crear incentivos contractualmente eficientes para asegurar la realización o intercambio de este conjunto de acciones y conocimientos productivos con valor de especificidad, y a la vez no contratables directamente, la 'propiedad compartida' puede proveer incentivos más eficientes que la opción de invertir, implementar o intercambiar dichas acciones y conocimientos mediante una empresa filial. Y ello por cuanto, existiendo alternativas 'sustitutas' de inversión y/o esfuerzos productivos de valor menos específico al nuevo negocio vitivinícola, los 'poderes de veto' que caracterizan a

48 The Economist, "Cured Meat. Feet in the though. The oldest method of preservation, and the best”, 23/12/2006 (pp. 88-90). 
un acuerdo de 'propiedad compartida' actúan como desincentivo a la realización de inversiones o esfuerzos de valor más general y que, por tanto, son menos valiosos para la oportunidad específica de creación de valor que se pretende implementar mediante el acuerdo tipo joint venture.

Sería deseable contrastar las ideas aquí sugeridas con evidencia empírica más detallada y que a la vez permita hacerse cargo, de forma más robusta, de otras posibles fuentes de influencia sobre la aparente relación que existiría entre la preferencia por acuerdos de 'propiedad compartida' y el objetivo de desarrollar proyectos de producción con significativo valor de especificidad y costos elevados de contratación. Un desafío relevante es el análisis de posibles efectos asociados a las escalas eficientes de operación en el caso de negocios vitivinícolas especializados en segmentos de mercado con elevado valor unitario del producto.

\section{ANEXO 1: \\ DATOS RECOPILADOS Y METODOLOGÍA APLICADA EN EL LEVANTAMIENTO DE ENCUESTAS}

En primer lugar se recurrió al Compendio Vitivinícola de Chile (Nuevos Mundos, 2004 y 2006), el cual contiene un catastro de viñas e identifican cuáles corresponden a JV y cuáles a propiedad 100\% extranjera. En este catastro se informa: nombre de la firma, localización, nombre del gerente y del enólogo, hectáreas plantadas, variedades de uva cultivada, denominación de origen, capacidad de bodega por tipo de infraestructura, cajas exportadas, rangos de precios de exportación, cantidad de países de destino, principales destinos de exportación y porcentaje exportado de la producción embotellada. Para completar datos faltantes se recurrió a datos de la Corporación Chilena del Vino, así como a diferentes trabajos y publicaciones sobre el sector (periódicamente la Corporación Chilena del Vino reúne toda publicación de diarios y revistas sobre el sector). Además se incluyó información entregada por cada empresa en sus respectivas páginas web.

En base a la información recabada se identificaron 40 firmas con participación extranjera en su propiedad que operan, o han operado, en la industria vitivinícola chilena. De este total, se obtuvieron datos completos para 32 viñas. En base a esta información se calcularon las exportaciones por empresa que se analizan en el texto central de este trabajo. Los cuadros A.1 y A.2 enumeran los JV y las filiales para los cuales se consiguió información. 


\section{CUADRO A.1: JOINT VENTURES VITIVINÍCOLAS (SOCIOS)}

\begin{tabular}{|c|c|c|c|c|}
\hline $\mathrm{N}^{\circ}$ & JV & Socia extranjera & Origen & Socia local \\
\hline 1 & Bodegas Conde de Aconcagua & González Byass & España & FH Engel \\
\hline 2 & Grand Bins Corpora Boisset & Flia. Boisset & Francia & $\begin{array}{l}\text { Corpora Vineyards and } \\
\text { Wineries }\end{array}$ \\
\hline 3 & La Reserva de Caliboro & Francesco Marone & Italia & Flia. Manzano \\
\hline 4 & Viña Almaviva & $\begin{array}{l}\text { Baron Philippe de } \\
\text { Rothschild-Mounton }\end{array}$ & Francia & Viña Concha y Toro \\
\hline 5 & Viña Altair & Chateau Dassault & Francia & Viña San Pedro \\
\hline 6 & Viña Aquitania & Bruno Prats y Paul Pontallier & Francia & Felipe de Solminihac \\
\hline 7 & Viña Caliterra & Robert Mondavi (Constellation) & USA & Viña Errázuriz \\
\hline 8 & Viña El Principal & Jean-Paul Valette & Francia & Hacienda El Principal \\
\hline 9 & Viña Los Vascos & $\begin{array}{l}\text { Domaines Barons de } \\
\text { Rothschild-Latife }\end{array}$ & Francia & Viña Santa Rita \\
\hline 10 & Viña Laroche \& Coderch & Michel Laroche & Francia & Jorge Coderch \\
\hline 11 & Viña Santa Eliana & $\begin{array}{l}\text { Viñedos del Jalón-Castillo } \\
\text { de Maluenda }\end{array}$ & España & Flia. Izquierdo-Saa \\
\hline 12 & Viña Seña & Robert Mondavi (Constellation) & USA & Viña Errázuriz \\
\hline 13 & Viña Terravid & Grupo Matarromera & España & Viña Portal del Alto \\
\hline 14 & Viña Veramonte & Canandaigua (Constellation) & USA & Flia. Huneeus \\
\hline 15 & Viña William Fèvre & Soc. Du Vignoble William Fèvre & Francia & Flia. Pino-Arrigorriaga \\
\hline 16 & Viña y Bodega Botalcura S.A. & Philippe Debrus y Jochen Döhle & Francia & Juan Fernando Waidele \\
\hline 17 & Viña Casa Lapostolle & Marnier-Lapostolle & Francia & Flia. Rabat \\
\hline
\end{tabular}

Fuente: Elaboración propia.

CUADRO A.2: $\quad$ FILIALES DE FIRMAS VITIVINÍCOLAS EXTRANJERAS

\begin{tabular}{llll}
\hline $\mathrm{N}^{\circ}$ & Filial & Propietario & Origen \\
\hline & & & \\
1 & Baron Philippe de & Baron Philippe de & Francia \\
& Rothschild Maipo SA & Rothschild-Mouton & \\
2 & Exportadora Calama Wines & George Strick y Lisa Denham & EE.UU. \\
3 & Inversiones Magnotta Chile Ltda. & Magnotta Winery Corporation & Canadá \\
4 & J\&F Lurton & J\&F Lurton & Francia \\
5 & Soc. Vinícola Miguel Torres & Bgas. Miguel Torres & España \\
6 & Viña Chateau Los Boldos & G. E. Massenez & Francia \\
7 & Viña Alempue & S/D & Alemania \\
8 & Viña Calina & Kendall Jackson & EE.UU. \\
9 & Viña Casa Donoso & Société Anonyme Les Vignobles & Francia \\
10 & Viña Odfjell & Dan Odfjell & Dinamarca \\
11 & Viña Tierra y Fuego & Lenz, Meler, Ruesch y Rufenacht Ltda & Alemania \\
12 & Viña Villard & Thierry Villard & Francia \\
13 & Viña Von Siebenthal & Mauro Von Siebenthal & Suiza \\
14 & Viña William Cole & William S Cole & EE.UU. \\
15 & Beringer Blass Wine & Beringer Blass Wine & EE.UU. \\
\hline
\end{tabular}

Fuente: Elaboración propia. 
Adicionalmente a la recopilación realizada de la literatura, se llevó a cabo un levantamiento de encuestas vía entrevistas con cada una de las firmas del listado elaborado. En la mayoría se entrevistó personalmente a gerentes de producción, enólogos y gerentes de comercialización de las firmas, mientras que el resto respondió al mismo cuestionario por correo electrónico. Al final del proceso se obtuvieron datos completos para 25 firmas con participación extranjera. Así, al referirnos a encuestas, la muestra contempla 25 firmas. El cuestionario utilizado se presenta a continuación.

ANEXO 2:

CUESTIONARIO PARA EMPRESAS VITIVINÍCOLAS

CON PARTICIPACIÓN EXTRANJERA

1. Año en que inicia actividades en el país.

2. Tamaño del emprendimiento local.

2.1. En término de has. cultivadas.

2.2. Cajas producidas por año.

3. ¿La viña efectúa contratos con otras viñas nacionales para el abastecimiento de uva?

4. ¿Utiliza la firma profesionales extranjeros en las siguientes áreas? (Indique cantidad en cada área)

4.1. Manejo de la viña y agronomía.

4.2. Manejo de bodega y enología.

4.3. Administración.

4.4. Comercialización.

5. La inversión extranjera consiste en:

5.1. Inversión completamente extranjera o filial.

5.2. Joint venture.

5.3. Otro tipo de asociación (en tal caso indique cuál).

6. En caso de asociación o joint venture, indique qué actividades cumple cada socio dentro de la misma. (En áreas de producción, administración y comercialización). 
7. En caso de asociación o joint venture, ¿Existen intercambios de profesionales entre la firma local y la firma extranjera, con fines de capacitación e intercambio de conocimiento?

ANEXO 3:

SOCIA DEL JV QUE IMPULSÓ ORIGINALMENTE EL ACUERDO DE PROPIEDAD COMPARTIDA

CUADRO A.3: $\quad$ JOINT VENTURES Y SOCIA IMPULSORA

\begin{tabular}{ll}
\hline JV & Socia impulsora \\
\hline Bodegas Conde de Aconcagua & Extranjera \\
Grand Vins Corpora Boisset & Extranjera \\
La Reserva de Caliboro & Extranjera \\
Viña Almaviva & S/D \\
Viña Altair & Extranjera \\
Viña Aquitania & Extranjera \\
Viña Caliterra & Extranjera \\
Viña El Principal & Local \\
Viña Los Vascos & Extranjera \\
Viña Laroche \& Coderch & Extranjera \\
Viña Santa Eliana & Extranjera \\
Viña Seña & Extranjera \\
Viña Terravid & Extranjera \\
Viña Veramonte & Local \\
Viña William Fèvre & Extranjera \\
Viña y Bodega Botalcura S.A. & Local \\
Viña Casa Lapostolle & Extranjera \\
\end{tabular}

Fuente: páginas webs de las empresas y entrevistas con personal de las viñas. S/ D: sin datos disponibles. 
ANEXO 4:

MAPA DE REGIONES VITIVINÍCOLAS CHILENAS

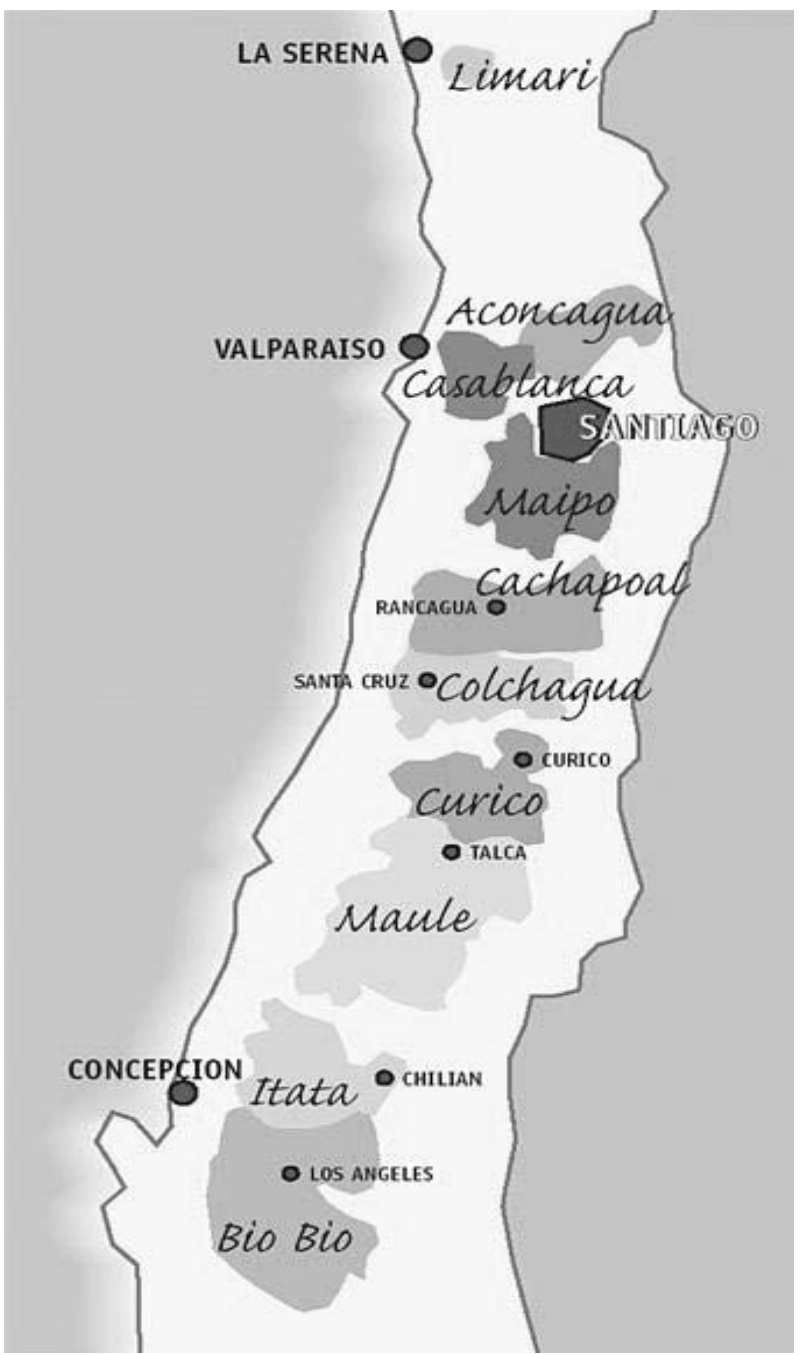

Fuente: www.protourschile.com (Protours Ltda.). 


\section{REFERENCIAS}

Agosin, M., E. Pastén y S. Vergara (2001): “Joint Ventures en la Industria Vitivinícola Chilena”. Mimeo, Universidad de Chile.

Aguirre, J. (2001): “El Sector Vitivinícola en la República Argentina a partir de 1990”. Mimeo, Instituto Nacional de Vitivinicultura.

Azpiazu, Daniel y Eduardo Basualdo (2003): Estudio Sectorial de la Industria Vitivinícola Argentina. Buenos Aires: FLACSO, $1^{\text {a }}$ edición.

Bajda, M. (2000): "Inversión Extranjera en la Industria Vitivinícola de Mendoza”. Mimeo, Universidad de Congreso.

Cai, H. (2003): “A Theory of Joint Asset Ownership”. En Rand Journal of Economics, 34, Spring, pp. 63-77.

Coelho, A. y A. de Sousa (2004): "Inter-Firm Relationships in the Beverage Industries: a Framework and Preliminary Findings”. Mimeo, XIV Congreso de Asociación Científica de Economía y Dirección de Empresas, Murcia, España.

Corporación Chilena del Vino (2003): Manual de Viticultura. Santiago: Universidad Santo Tomás, $1^{a}$ edición. - (2005): "Información Estadística”, www.ccv.cl

Del Pozo, J. (1998): Historia del Vino Chileno. Desde 1850 Hasta Hoy. Santiago: Editorial Universitaria, $1^{\text {a }}$ edición.

El Mercurio (2005): “El Peso de los Vinos Soñados”. Economía y Negocios, 19 de junio. (2006a): "El Temporero es Rey”. Economía y Negocios, 27 de marzo. - (2006b): "Vinos de Etiqueta”. Revista Wikén, 23 de junio. - (2006c): “No Somos una Industria, Somos Agricultores”. Revista del Campo, 21 de agosto. (2007). 11 de marzo de 2007.

Farinelli, F. (2003): "Technological Catch-Up and Learning Dynamics in the Chilean Wine Industry”. Mimeo, UNCTAD.

Garner, B. (2004): Black's Law Dictionary. Ed. Thompson-West, 8 a edición.

Giuliani, E. (2002): “The Co-evolution of Tacit and Codified Knowledge: Some Implications on the Competitiveness of Traditional and Emerging Countries in the Wine Industry”. Mimeo, División de Desarrollo Productivo y Empresarial, CEPAL, Santiago de Chile.

Giuliani, E. y M. Bell (2005): "The Micro-Determinants of Meso-Level Learning and Innovation: Evidence from a Chilean Wine Cluster”. En Research Policy, 34 (1), 47-68.

Grossman S. y O. Hart (1986): "The Costs and Benefits of Ownership: A Theory of Vertical and Lateral Integration”. En Journal of Political Economy, 94 (4), pp. 691-719.

Halonen, M. (2002): "Reputation and the Allocation of Ownership". En The Economic Journal, 112 (481), pp. 539-558.

Hart, Oliver (1995): Firms, Contracts and Financial Structure. Oxford University Press, $1^{\text {st }}$ edition.

Hart, O. y J. Moore (1990): “Property Rights and the Nature of the Firm”. En Journal of Political Economy, 98 (6), pp. 1119-1158.

Holmstrom, B. y J. Roberts (1998): “The Boundaries of the Firm Revisited”. En Journal of Economic Perspectives, 12 (4), pp. 73-94. 
http://www.chilevinos.cl: “El Dilema de los Íconos”, 13 de marzo, 2006.

http://www.latife.com.

http://www.mondavi.com.

http://www.protourschile.com. (Protours Ltda.).

Klein, B., R. Crawford y A. Alchain (1978): "Vertical Integration, Appropriable Rents, and the Competitive Contracting Process”. En Journal of Law and Economics, 21, pp. 297-326.

La Segunda (2005): “Nuevas Regiones Vitivinícolas”. Economía, 3 de mayo.

Mac Cawley, A. (2006): "Estudio de los Joint Ventures en Viñas Chilenas: ¿Éxitos o Fracasos? Mimeo, Departamento de Agronomía, Pontificia Universidad Católica de Chile, Santiago.

Mo, F. (1979): “Vitivinicultura. Mito. Leyenda. Historia”. Buenos Aires: Ed. De Palma.

Morton, F. y J. Podolny (1998): "Love or Money? The Effects of Owner Motivation in the California Wine Industry”. National Bureau of Economic Research, Working Paper 6783.

Nuevos Mundos (2004): Compendio Vitivinícola de Chile. Santiago: Editorial Nuevos Mundos, Santiago, Chile, $1^{\text {a }}$ edición.

(2006): Compendio Vitivinícola de Chile. Santiago: Editorial Nuevos Mundos, $1^{\mathrm{a}}$ edición.

Polanyi, M. (1967): The Tacit Dimension. Anchor Books, Doubleday \& Co, $1^{\mathrm{a}}$ edición.

Schamel, G. (2000): "Individual and Collective Reputation Indicators of Wine Quality". CIES, Discussion Paper 00/09, University of Adelaide, marzo.

The Economist (1998): "European Wine. Put a Cork in It”, 27 de junio.

- (1999): “The Globe in a Glass”, 18 de diciembre. (2000): “Australian Wine. In Search of Icons”, 24 de junio.

(2006): "Cured Meat. Feet in the Though. The Oldest Method of Preservation, and the Best”. 23/12/2006.

Troncoso, J., J. Riquelme, F. Laurie y J. Abarca (2002): "Evaluación de las Ventajas Relativas de la Vendimia Mecanizada en Chile Central”. En Agricultura Técnica, 62, (2).

Unwin, T. (1991): Wine and the Vine: an Historical Geography of Viticulture. Routledge, $1^{\mathrm{a}}$ edición.

Vendimia (revista de la Corporación Chilena del Vino). 2002.

Vergara, S. (2001): “El Mercado Vitivinícola Mundial y el Flujo de Inversión Extranjera en Chile”. En Serie Desarrollo Productivo, CEPAL, Santiago de Chile. 